\title{
PROOF OF TAYLOR'S CONJECTURE ON MAGNETIC HELICITY CONSERVATION
}

\author{
DANIEL FARACO AND SAULI LINDBERG
}

\begin{abstract}
We prove Taylor's conjecture which says that in 3D MHD, magnetic helicity is conserved in the ideal limit in bounded, simply connected, perfectly conducting domains. When the domain is multiply connected, magnetic helicity depends on the vector potential of the magnetic field. In that setting we show that magnetic helicity is conserved for a large and natural class of vector potentials but not in general for all vector potentials. As an analogue of Taylor's conjecture in $2 \mathrm{D}$, we show that mean square magnetic potential is conserved in the ideal limit, even in multiply connected domains.
\end{abstract}

\section{INTRODUCTION}

Magnetohydrodynamics (MHD in short) couples Navier-Stokes equations with Maxwell's equations to study the macroscopic behaviour of electrically conducting fluids such as plasmas and liquid metals (see [26] and 44]). Given a domain $\Omega \subset \mathbb{R}^{3}$ and a time interval $[0, T)$, the Cauchy problem for the incompressible, viscous, resistive $M H D$ equations consists of the equations

$$
\begin{aligned}
& \partial_{t} u+(u \cdot \nabla) u-(b \cdot \nabla) b-\nu \Delta u+\nabla \Pi=0, \\
& \partial_{t} b+\operatorname{curl}(b \times u)+\mu \operatorname{curl} \operatorname{curl} b=0, \\
& \operatorname{div} u=\operatorname{div} b=0, \\
& u(\cdot, 0)=u_{0}, b(\cdot, 0)=b_{0},
\end{aligned}
$$

where $b$ is the magnetic field, $u$ is the velocity field, $\Pi$ is the total pressure, $\nu>0$ is the coefficient of viscosity, $\mu>0$ is the coefficient of resistivity and the initial datas $u_{0}$ and $b_{0}$ are divergence-free. The Navier-Stokes equations are a special case of MHD where $b \equiv 0$. Furthermore, setting $\mu=\nu=0$ one obtains the ideal MHD equations, while in the case $\mu=0<\nu, 1.1-1.3$ are called the non-resistive $M H D$ equations.

In this work we consider Leray-Hopf solutions of $1.1-1.4$ in a bounded domain $\Omega$ of $\mathbb{R}^{3}$ that has a $\mathscr{C}^{1,1}$ boundary $\Gamma$. As we want to incorporate Tokamaks and other laboratory plasma configurations, it is mandatory to consider multiply connected domains (see Assumptions 2.1 2.2 for the exact conditions on $\Omega$ ). We use the

2010 Mathematics Subject Classification. 35Q35, 76W05, 76B03.

Key words and phrases. Magnetohydrodynamics, magnetic helicity, mean-square magnetic potential, Taylor conjecture, compensated compactness.

D.F. was partially supported by ICMAT Severo Ochoa projects SEV-2011-0087 and SEV2015-556, the grants MTM2014-57769-P-1 and MTM2014-57769-P-3 (Spain) and the ERC grant 307179-GFTIPFD. S.L. was supported by the ERC grant 307179-GFTIPFD. 
standard no-slip and perfect conductivity boundary conditions

$$
\begin{aligned}
& \left.u\right|_{\Gamma}=0, \\
& \left.b \cdot n\right|_{\Gamma}=0 \quad \text { and } \quad(\operatorname{curl} b) \times\left. n\right|_{\Gamma}=0,
\end{aligned}
$$

(see $\$ 2.4$ for precise definitions).

The existence of Leray-Hopf solutions in smooth simply connected domains goes back to [16] and [44], and in [51, existence is shown under the slip without friction conditions on $u$. The more complicated case of smooth multiply connected domains is covered in the doctoral dissertation [31. Since 31] is not readily available, we present our version of the proof for $\mathscr{C}^{1,1}$ multiply connected domains in the Appendix. For local-in-time existence and uniqueness of strong solutions as well as weak solutions in suitable Besov spaces for ideal MHD see 35, [42 and [43, and for the case of non-resistive MHD see [10, [20, [21] and [33. For further references see [26, p. 57].

In ideal 3D MHD, smooth solutions conserve the total energy $2^{-1} \int_{\Omega}\left(|u(x, t)|^{2}+\right.$ $\left.|b(x, t)|^{2}\right) d x$ and the cross helicity $\int_{\Omega} u(x, t) \cdot b(x, t) d x$ in time. In simply connected domains the magnetic helicity

$$
\int_{\Omega} \psi(x, t) \cdot b(x, t) d x
$$

where $\psi$ is a vector potential of $b$ (that is, $\operatorname{curl} \psi=b$ ), is also conserved by smooth solutions and is independent of the choice of $\psi$.

Recently obtained numerical evidence points, however, towards anomalous energy dissipation, that is, the rate of total energy dissipation in viscous, resistive MHD does not tend to zero when $\mu, \nu \rightarrow 0$ (when the Reynolds number and magnetic Reynolds number tend to infinity); see [13, 34] and 36. Thus, if ideal MHD equations are to be a good model for magnetohydrodynamic turbulence at very high Reynolds number and magnetic Reynolds number, then the equations must possess (physically realistic) energy dissipative solutions. This is in analogy to the celebrated Onsager Conjecture on Euler equations (see 7, [12, 17, 29 and 39]). In ideal MHD, bounded non-vanishing weak solutions with compact support in time (thus violating total energy conservation) were found in [5, while non-vanishing smooth strict subsolutions with compact support in space-time were constructed in 18 .

In stark contrast to total energy, magnetic helicity has proved to be a very robust time invariant of ideal MHD. First, Caflisch, Klapper and Steele showed in [8] that magnetic helicity is conserved whenever $u \in C\left([0, T] ; B_{3, \infty}^{\alpha_{1}}\left(\mathbb{T}^{3}, \mathbb{R}^{3}\right)\right)$ and $b \in$ $C\left([0, T] ; B_{3, \infty}^{\alpha_{2}}\left(\mathbb{T}^{3}, \mathbb{R}^{3}\right)\right)$ with $\alpha_{1}+2 \alpha_{2}>0$, and next Kang and Lee showed magnetic helicity conservation for $u, b \in C_{w}\left([0, T] ; L^{2}\left(\mathbb{T}^{3}, \mathbb{R}^{3}\right)\right) \cap L^{3}\left(0, T ; L^{3}\left(\mathbb{T}^{3}, \mathbb{R}^{3}\right)\right)$ in 30. In [18, the authors extended conservation to subsolutions and weak limits of solutions in $L^{3}\left(0, T ; L^{3}\left(\mathbb{T}^{3}, \mathbb{R}^{3}\right)\right)$.

It is still open whether magnetic helicity is conserved if $u$ and $b$ belong to the physically natural energy space $L^{\infty}\left(0, T ; L^{2}\left(\mathbb{T}^{3}, \mathbb{R}^{3}\right)\right)$. However, a straightforward adaptation of our Theorem 1.2 to the torus implies that conservation occurs if $u, b \in L^{\infty}\left(0, T ; L^{2}\left(\mathbb{T}^{3}, \mathbb{R}^{3}\right)\right)$ are a weak ideal limit of Leray-Hopf solutions (see Definition 1.1 and Corollary 1.3 , which is arguably the physically relevant case. 
It has been conjectured in the physics literature that magnetic helicity is approximately conserved at very low resistivities (see [45] where the conjecture was first formulated by Taylor). Mathematically, the conjecture says that magnetic helicity is conserved in the ideal limit $\mu, \nu \rightarrow 0$ (see [8, p. 444]). Taylor's conjecture has been successful in explaining magnetic structures in laboratory plasmas, e.g., in the prediction of the relaxed state of a reversed field pinch, and lies at the heart of Taylor relaxation theory (for reviews with numerous further references see [6] and [46]).

In Theorem 1.2 we prove Taylor's conjecture under weak and natural assumptions. We consider arbitrary weak limits of Leray-Hopf solutions when $\mu_{j}, \nu_{j} \rightarrow 0$ (which exist, up to a subsequence, whenever the $L^{2}$ norms of the initial datas are uniformly bounded). In particular, we do not assume that the weak limits satisfy the ideal MHD equations. Recall that

$$
L_{\sigma}^{2}\left(\Omega, \mathbb{R}^{3}\right):=\left\{v \in L^{2}\left(\Omega, \mathbb{R}^{3}\right): \operatorname{div} v=0,\left.v \cdot n\right|_{\Gamma}=0\right\} .
$$

Definition 1.1. Given Leray-Hopf solutions $\left(u_{j}, b_{j}\right)$ of 1.1 1.6 with $\mu_{j}, \nu_{j}>0$ and initial datas $u_{j, 0}, b_{j, 0} \in L_{\sigma}^{2}\left(\Omega, \mathbb{R}^{3}\right)$ suppose that $\mu_{j}, \nu_{j} \rightarrow 0$ and that $u_{j, 0} \rightarrow u_{0}$ and $b_{j, 0} \rightarrow b_{0}$ in $L_{\sigma}^{2}\left(\Omega, \mathbb{R}^{3}\right)$. Assume that $u_{j} \stackrel{*}{\rightarrow} u$ and $b_{j} \stackrel{*}{\rightarrow} b$ in $L^{\infty}\left(0, T ; L_{\sigma}^{2}\left(\Omega, \mathbb{R}^{3}\right)\right)$. We then say that $(u, b)$ is a weak ideal limit of $\left(u_{j}, b_{j}\right)$.

If instead $\mu_{j} \rightarrow 0$ and $\nu_{j}=\nu>0$ for every $j \in \mathbb{N}$, we say that $(u, b)$ is a weak non-resistive limit of $\left(u_{j}, b_{j}\right)$.

Taylor's conjecture concerns the case where magnetic helicity is gauge invariant (i.e. independent of the choice of the vector potential of $b$ ), that is, simply connected domains. The following theorem proves Taylor's conjecture.

Theorem 1.2. Suppose $\Omega$ is simply connected and $(u, b)$ is a weak ideal limit of Leray-Hopf solutions $\left(u_{j}, b_{j}\right)$ with $\mu_{j}, \nu_{j} \rightarrow 0$. Then $\int_{\Omega} \psi(x, t) \cdot b(x, t) d x$ is a.e. constant in $t$ for every vector potential $\psi \in L^{\infty}\left(0, T ; W^{1,2}\left(\Omega, \mathbb{R}^{3}\right)\right)$ of $b$.

Although in Theorem 1.2 we do not assume that $u$ and $b$ satisfy the ideal MHD equations, we present a corollary on solutions of ideal MHD. If a solution $(u, b)$ lies in the energy space $L^{\infty}\left(0, T ; L_{\sigma}^{2}\left(\Omega, \mathbb{R}^{3}\right)\right)$, then we may choose representatives $u, b \in C_{w}\left([0, T) ; L_{\sigma}^{2}\left(\Omega, \mathbb{R}^{3}\right)\right)$; this can be proved by slightly modifying [24, Lemmas $2.1-2.2]$.

Corollary 1.3. Suppose $\Omega$ is simply connected and $u, b \in C_{w}\left([0, T) ; L_{\sigma}^{2}\left(\Omega, \mathbb{R}^{3}\right)\right)$ form a weak solution of ideal MHD. If $(u, b)$ is a weak ideal limit of Leray-Hopf solutions $\left(u_{j}, b_{j}\right)$, then $b$ conserves magnetic helicity in time.

While simply connected domains (and especially the torus $\mathbb{T}^{3}$ ) allow a relatively neat mathematical treatment, we also cover multiply connected domains in order to incorporate plasma containers in typical laboratory settings. The topology of multiply connected domains leads, however, to mathematical complications starting with the very definition of magnetic helicity.

Consider an arbitrary weak ideal limit $(u, b)$ of Leray-Hopf solutions $\left(u_{j}, b_{j}\right)$. If the domain $\Omega$ is multiply connected, then $\int_{\Omega} \psi(x, t) \cdot b(x, t) d x$ depends on the choice of the vector potential $\psi$. The basic reason behind this gauge dependence is the fact that when $\Omega$ is multiply connected, the orthogonal complement of ker(curl) in $L^{2}\left(\Omega, \mathbb{R}^{3}\right)$ is a strict subspace of $L_{\sigma}^{2}\left(\Omega, \mathbb{R}^{3}\right)$ - in other words, the set of harmonic 
Neumann vector fields

$$
L_{H}^{2}\left(\Omega, \mathbb{R}^{3}\right):=\left\{v \in L_{\sigma}^{2}\left(\Omega, \mathbb{R}^{3}\right): \operatorname{curl} v=0\right\}
$$

is non-empty. For a physical interpretation of $L_{H}^{2}\left(\Omega, \mathbb{R}^{3}\right)$ see e.g. [9, pp. 428-430]. We write

$$
L_{\sigma}^{2}\left(\Omega, \mathbb{R}^{3}\right)=L_{\Sigma}^{2}\left(\Omega, \mathbb{R}^{3}\right) \oplus L_{H}^{2}\left(\Omega, \mathbb{R}^{3}\right)
$$

a useful intrinsic characterisation of $L_{\Sigma}^{2}\left(\Omega, \mathbb{R}^{3}\right)$ was given in 23 (see Theorem 3.2). For the purposes of this article, it is also illuminating to use a characterisation familiar from Hodge-Friedrichs-Morrey decomposition theory (see [37]),

$$
L_{\Sigma}^{2}\left(\Omega, \mathbb{R}^{3}\right)=\left\{\operatorname{curl} \psi: \psi \in W^{1,2}\left(\Omega, \mathbb{R}^{3}\right), \psi \times\left. n\right|_{\Gamma}=0\right\} .
$$

In fact, we will need slightly more refined versions of (1.8), see Theorem 3.8 and Remark 3.9 .

Bearing in mind (1.7), we decompose $b$ uniquely as

$$
b=b_{\Sigma}+b_{H} \quad\left(b_{\Sigma} \in L^{\infty}\left(0, T ; L_{\Sigma}^{2}\left(\Omega, \mathbb{R}^{3}\right)\right) \text { and } b_{H} \in L^{\infty}\left(0, T ; L_{H}^{2}\left(\Omega, \mathbb{R}^{3}\right)\right)\right)
$$

and use similar notation for every $b_{j}$. In multiply connected domains, we prove that $\int_{\Omega} \psi(x, t) \cdot b(x, t) d x$ is conserved for all vector potentials $\psi \in L^{\infty}\left(0, T ; W^{1,2}\left(\Omega, \mathbb{R}^{3}\right)\right)$ of $b$ if and only if the harmonic part $b_{H}=0$. There exist, however, weak ideal limits $(u, b)$ of Leray-Hopf solutions with $b_{H} \neq 0$ (see Proposition 3.5 for both claims).

We are thus led to the following question in multiply connected domains:

$$
\text { Is } \int_{\Omega} \psi(x, t) \cdot b(x, t) d x \text { conserved for some natural class of potentials } \psi \text { ? }
$$

We give a positive answer to 1.10 in Corollary 1.6 . First, in Theorem 1.5 we compute the magnetic helicity dissipation rate for arbitrary Leray-Hopf solutions and arbitrary vector potentials. In (1.11) we are able to compute the dissipation rate also for weak ideal limits and all their potentials. Corollary 1.6 then gives a condition on potentials that is coherent with (1.8) and yields magnetic helicity conservation.

We use the decomposition in $(1.9)$ in order to give a formula for the time evolution of magnetic helicity. The components $b_{\Sigma}$ and $b_{H}$ of $b$ behave in rather differing ways; in particular, $b_{H}$ is constant in time (see Proposition 4.3). Because of difficulties described in 4.1 we also need to decompose $\psi$ in order to take advantage of the different features of $b_{\Sigma}$ and $b_{H}$ :

$$
\psi=\psi^{\Sigma}+\psi^{H} \quad\left(\operatorname{curl} \psi^{\Sigma}=b_{\Sigma} \text { and } \operatorname{curl} \psi^{H}=b_{H}\right) .
$$

The decomposition $\psi=\psi^{\Sigma}+\psi^{H}$ is not unique, and a judicious choice of the components $\psi^{\Sigma}, \psi^{H} \in L^{\infty}\left(0, T ; W^{1,2}\left(\Omega, \mathbb{R}^{3}\right)\right)$ is a fundamental part of the proof of Theorem 1.5. In fact, we end up performing a further decomposition of $\psi^{\Sigma}$, and the whole decomposition of $\psi$ is described in $\$ 3.2$ and $\$ 4.1$.

In order to state Theorem 1.5 we already note below that given $\psi$, there exists a canonical choice of $\psi^{H}$, and we use it for all the vector potentials in this article. In particular, with this choice, $\partial_{t} b_{H}=0$ implies that $\partial_{t} \psi^{H}=0$.

Definition 1.4. Suppose that $v=v_{\Sigma}+v_{H} \in L^{\infty}\left(0, T ; L_{\sigma}^{2}\left(\Omega, \mathbb{R}^{3}\right)\right)$ and that $\psi \in$ $L^{\infty}\left(0, T ; W^{1,2}\left(\Omega, \mathbb{R}^{3}\right)\right)$ satisfies curl $\psi=v$. We denote by $\psi^{H}$ the unique mapping in $L^{\infty}\left(0, T ; W^{1,2}\left(\Omega, \mathbb{R}^{3}\right) \cap L_{\Sigma}^{2}\left(\Omega, \mathbb{R}^{3}\right)\right)$ such that $\operatorname{curl} \psi^{H}=b_{H}$ (see Theorem 3.7), and we furthermore denote $\psi^{\Sigma}:=\psi-\psi^{H}$. 
We are now in a position to state our main theorem; the strategy of the proof is described in 4.14 .2 and the details are presented in 4.34 .6 .

Theorem 1.5. Suppose a domain $\Omega \subset \mathbb{R}^{3}$ satisfies Assumption 2.1, and assume that $(u, b)$ is a weak ideal limit or weak resistive limit of Leray-Hopf solutions $\left(u_{j}, b_{j}\right), j \in \mathbb{N}$. Then any vector potentials $\psi_{j}$ and $\psi_{j, 0}$ of $b_{j}$ and $b_{j, 0}$ satisfy

$$
\begin{aligned}
\int_{\Omega} \psi_{j}(x, t) \cdot b_{j}(x, t) d x & =\int_{\Omega} \psi_{j, 0}(x) \cdot b_{j, 0}(x) d x \\
& -2 \mu_{j} \int_{0}^{t} \int_{\Omega} b_{j}(x, \tau) \cdot \operatorname{curl} b_{j}(x, \tau) d x d \tau \\
& -\int_{\Gamma}\left[\psi_{j}^{\Sigma}(x, t)-\psi_{j, 0}^{\Sigma}(x)\right] \times n \cdot \psi_{j, 0}^{H}(x) d x
\end{aligned}
$$

for all $j \in \mathbb{N}$ and $t \in[0, T)$. Furthermore,

$$
\int_{\Omega} \psi(x, t) \cdot b(x, t) d x=\int_{\Omega} \psi_{0}(x) \cdot b_{0}(x) d x-\int_{\Gamma}\left[\psi^{\Sigma}(x, t)-\psi_{0}^{\Sigma}(x)\right] \times n \cdot \psi_{0}^{H}(x) d x
$$

for a.e. $t \in(0, T)$ and all vector potentials $\psi$ and $\psi_{0}$ of $b$ and $b_{0}$.

Formula (1.11) allows us to show magnetic helicity conservation for a large class of vector potentials. The class is specified in 1.12 , and its naturality is apparent from 1.8 and 1.11 .

Corollary 1.6. Suppose the assumptions of Theorem 1.5 hold. If

$$
\psi_{j}^{\Sigma} \times\left. n\right|_{\Gamma}=\psi^{\Sigma} \times\left. n\right|_{\Gamma}=0 \quad \text { and } \quad \psi_{j, 0}^{\Sigma} \times\left. n\right|_{\Gamma}=\psi_{0}^{\Sigma} \times\left. n\right|_{\Gamma}=0,
$$

then

$$
\int_{\Omega} \psi(x, t) \cdot b(x, t) d x=\int_{\Omega} \psi_{0}(x) \cdot b_{0}(x) d x=\lim _{j \rightarrow \infty} \int_{\Omega} \psi_{j, 0}(x) \cdot b_{j, 0}(x) d x
$$

for a.e. $t \in(0, T)$. In particular, under condition 1.12 , the magnetic helicity of $b$ is independent of the choice of $\psi$.

In $\$ 5$ we prove a two-dimensional analogue of Theorem 1.5 in bounded, multiply connected Lipschitz domains, mean-square magnetic potential is conserved in the weak ideal limit. In 2D, there exists a canonical choice of potentials, and so we can follow the philosophy of [18] which is based on $\mathcal{H}^{1}$-BMO duality and compensated compactness. In fact, we also show that in multiply connected domains, all solutions of ideal MHD in the energy space conserve magnetic mean-square potential, extending a similar result on the torus $\mathbb{T}^{2}$ from [18.

In three dimentions, when magnetic field lines are allowed to cross $\Gamma$, that is, the assumption $\left.b \cdot n\right|_{\Gamma}=0$ is dropped, magnetic helicity is no longer gauge invariant even for smooth solutions of ideal MHD in simply connected domains. In such a setting the so-called relative magnetic helicity, defined in [3] and [22], can be studied instead. We defer a treatment of relative magnetic helicity to a subsequent work.

\section{BACKGROUND}

In this chapter we review tools and results needed in this article. We first fix our assumptions on the domain $\Omega$ in $\$ 2.1$ and recall basic material on boundary traces of Sobolev and $L^{p}$ functions in $\$ 2.2$ \$2.3 reviews some standard results on 
time-dependent mappings in Bochner spaces, and in 2.4 we discuss Leray-Hopf solutions of viscous, resistive 3D MHD equations and the notion of inviscid, nonresistive limit.

2.1. Assumptions on the domain. We start by fixing our assumptions on the domain $\Omega$, and we illustrate the assumptions in Figure 1. Our exposition follows [1. pp. 835-836] (see also [47]).

Assumption 2.1. The domain $\Omega \subset \mathbb{R}^{3}$ is bounded and its boundary $\Gamma$ is of class $\mathscr{C}^{1,1}$ and has a finite number of connected components denoted by $\Gamma_{1}, \ldots, \Gamma_{K}$.

Another assumption is introduced in order to produce a simply connected domain by making cuts into $\Omega$. The cuts will, however, only play an implicit role in this article.

Assumption 2.2. There exist connected open surfaces $\Sigma_{j}, 1 \leq j \leq N$, contained in $\Omega$ and satisfying the following conditions:

(i) Each surface $\Sigma_{j}$ is an open subset of a smooth manifold $\mathscr{M}_{j}$.

(ii) The boundary of each $\Sigma_{j}$ is contained in $\partial \Omega$.

(iii) $\bar{\Sigma}_{i} \cap \bar{\Sigma}_{j}=\emptyset$ whenever $i \neq j$.

(iv) The open set $\dot{\Omega}:=\Omega \backslash \sup _{j=1}^{N} \Sigma_{j}$ is simply connected and pseudo-Lipschitz (see Definition 2.3 below).

The sets $\Sigma_{j}$ are called cuts.

The notion of a pseudo-Lipschitz domain is a generalization of a Lipschitz domain that allows the domain to locally lie on both sides of its boundary.

Definition 2.3. A bounded domain $\Delta \subset \mathbb{R}^{3}$ is called pseudo-Lipschitz if for every $x \in \partial \Delta$ there exists an integer $r(x) \in\{1,2\}$ and a radius $\rho_{0}>0$ such that whenever $0<\rho<\rho_{0}$, the intersection $\Delta \cap B(x, \rho)$ has $r(x)$ connected components, each one with a Lipschitz boundary.

Assumptions 2.1 2.2 are standard in the study of fluid dynamics in multiply connected domains (see e.g. [1], 23] and [47]) and will remain in place for the rest of this article (except $\$ 5$ where we discuss the two-dimensional setting). In particular, a solid torus clearly satisfies Assumptions 2.1 2.2.

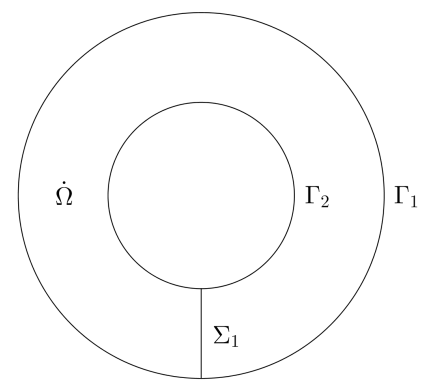

(A) Projection of a torus $\Omega \subset \mathbb{R}^{3}$ into the $x y$-plane.

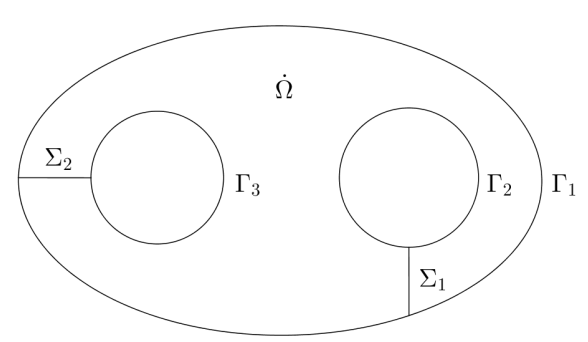

(B) Projection of a double torus into the $x y$-plane.

FiguRE 1 
2.2. Traces of Sobolev functions. We recall results on boundary traces, normal traces and tangential traces and refer to [25, 27] and [38, for the proofs. In Theorems 2.4 2.7 the assumption that $\Gamma$ is $\mathscr{C}^{1,1}$ can in fact be relaxed to $\Gamma$ being Lipschitz regular. The first trace theorem we present is a special case of [25, Theorem II.4.1].

Theorem 2.4. Let $1 \leq p<\infty$. Then there exists a unique, continuous linear map $\gamma: W^{1, p}(\Omega) \rightarrow L^{p}(\Gamma)$ such that for every $u \in C^{\infty}(\bar{\Omega})$ we have $\gamma(u)=u$ on $\Gamma$.

We denote by $W^{1-1 / p, p}(\Gamma)$ the subspace of $L^{p}(\Gamma)$ of functions for which

$$
\|u\|_{W^{1-1 / p, p}(\Gamma)}:=\|u\|_{L^{p}(\Omega)}+\left(\int_{\Gamma} \int_{\Gamma} \frac{|u(x)-u(y)|^{p}}{|x-y|^{1+p}} d S(x) d S(y)\right)^{\frac{1}{p}}<\infty .
$$

The space $W^{1-1 / p, p}(\Gamma)$ is dense in $L^{p}(\Gamma)$ and complete in the norm $\|\cdot\|_{W^{1-1 / p, p}(\Gamma)}$. When $1<p<\infty$, the trace operator $\gamma$ is a Banach space isomorphism from the quotient space $W^{1, p}(\Omega) / W_{0}^{1, p}(\Omega)$ onto $W^{1-1 / p, p}(\Gamma)$ (see $[38, \S 2.5$, Theorems 5.5 and 5.7]):

Theorem 2.5. Let $1<p<\infty$. If $u \in W^{1, p}(\Omega)$, then $\gamma(u) \in W^{1-1 / p, p}(\Gamma)$ and

$$
\|\gamma(u)\|_{W^{1-1 / p, p}(\Gamma)} \lesssim \Omega, p\|u\|_{W^{1, p}(\Omega)} .
$$

Conversely, given $w \in W^{1-1 / p, p}(\Gamma)$ there exists $u \in W^{1, p}(\Omega)$ such that $\gamma(u)=w$ and $\|u\|_{W^{1, p}(\Omega)} \lesssim \Omega, p\|\gamma(u)\|_{W^{1-1 / p, p}(\Gamma)}$.

For convenience we will denote the trace $\gamma(u)$ simply by $u$. Whenever $u \in$ $C^{\infty}\left(\bar{\Omega}, \mathbb{R}^{3}\right)$, the normal trace $u \cdot n$ and the tangential trace $u \times n$ are well-defined on the boundary $\Gamma$ and the generalized Gauss identity and Green's formula

$$
\langle u \cdot n, \varphi\rangle_{\Gamma}=\int_{\Omega} u(x) \cdot \nabla \varphi(x) d x+\int_{\Omega} \varphi(x) \operatorname{div} u(x) d x, \quad \varphi \in W^{1, p^{\prime}}(\Omega)
$$

$$
\langle u \times n, \psi\rangle_{\Gamma}=\int_{\Omega} \operatorname{curl} u(x) \cdot \psi(x) d x-\int_{\Omega} u(x) \cdot \operatorname{curl} \psi(x) d x, \quad \psi \in W^{1, p^{\prime}}\left(\Omega, \mathbb{R}^{3}\right)
$$

hold, where $\langle u \cdot n, \varphi\rangle_{\Gamma}$ and $\langle u \times n, \psi\rangle_{\Gamma}$ are standard surface integrals (but can also be interpreted in terms of $W^{-1 / p, p}(\Gamma)-W^{1-1 / p^{\prime}, p^{\prime}}(\Gamma)$ duality).

Normal and tangential traces are extended to the function spaces defined next: when $1<p<\infty, H^{p}(\operatorname{div}, \Omega):=\left\{v \in L^{p}\left(\Omega, \mathbb{R}^{3}\right): \operatorname{div} v \in L^{p}(\Omega)\right\}$ is endowed with the norm $\|v\|_{H^{p}(\operatorname{div}, \Omega)}:=\left(\|v\|_{L^{p}(\Omega)}^{p}+\|\operatorname{div} v\|_{L^{p}(\Omega)}^{p}\right)^{1 / p}$, while $H^{p}(\operatorname{curl}, \Omega):=$ $\left\{v \in L^{p}\left(\Omega, \mathbb{R}^{3}\right): \operatorname{curl} v \in L^{p}\left(\Omega, \mathbb{R}^{3}\right)\right\}$ is given the norm $\|v\|_{H^{p}(\operatorname{curl}, \Omega)}:=\left(\|v\|_{L^{p}(\Omega)}^{p}+\right.$ $\left.\|\operatorname{curl} v\|_{L^{p}(\Omega)}^{p}\right)^{1 / p}$.

Theorem 2.6. Suppose $1<p<\infty$. Then the normal trace has a unique bounded extension $u \mapsto u \cdot n: H^{p}(\operatorname{div}, \Omega) \rightarrow W^{-1 / p, p}(\Gamma)$ and the generalized Gauss identity (2.1) holds.

For a proof of Theorem 2.6 see [25, Theorem III.2.2]. In a similar vein, a tangential trace is well-defined whenever $v \in L^{p}\left(\Omega, \mathbb{R}^{3}\right)$ and $\operatorname{curl} v \in L^{p}\left(\Omega, \mathbb{R}^{3}\right)$ :

Theorem 2.7. Suppose $1<p<\infty$. Then the tangential trace has a unique bounded extension $u \mapsto u \times n: H_{p}(\operatorname{curl} ; \Omega) \rightarrow W^{-1 / p, p}\left(\Gamma, \mathbb{R}^{3}\right)$ and the generalized Green's formula (2.2) holds. 
Finally we mention a characterisation of $W^{1,2}\left(\Omega, \mathbb{R}^{3}\right)$ by Foias and Temam (see e.g. [27, Corollary 3.7]). Here Lipschitz continuity of $\Gamma$ would not be sufficient (see [1, p. 832]).

Theorem 2.8. We have $W^{1,2}\left(\Omega, \mathbb{R}^{3}\right)=\left\{v \in L^{2}\left(\Omega, \mathbb{R}^{3}\right)\right.$ : $\operatorname{div} v \in L^{2}(\Omega)$, curl $v \in$ $\left.L^{2}\left(\Omega, \mathbb{R}^{3}\right), v \cdot n \in W^{1 / 2,2}(\Gamma)\right\}$ and

$$
\|v\|_{W^{1,2}(\Omega)} \lesssim \Omega\|v\|_{L^{2}(\Omega)}+\|\operatorname{div} v\|_{L^{2}(\Omega)}+\|\operatorname{curl} v\|_{L^{2}(\Omega)}+\|v \cdot n\|_{W^{1 / 2,2}(\Gamma)}
$$

for all $v \in W^{1,2}\left(\Omega, \mathbb{R}^{3}\right)$.

2.3. Bochner spaces. We recall some basic facts on time-dependent mappings in Bochner spaces in a generality needed in this article. We do not discuss the definitions of Bochner measurability and Bochner integrability but refer to [28] for a thorough introduction to Bochner spaces and to [41] for a shorter one with an emphasis on applications in PDE's.

Whenever $1 \leq p<\infty$ and $X$ is a Banach space, the Bochner space $L^{p}(0, T ; X)$ consists of (classes with respect to equality a.e. $t \in(0, T)$ of) Bochner integrable functions $v:(0, T) \rightarrow X$ satisfying $\int_{0}^{T}\|v(\cdot, t)\|_{X}^{p} d t<\infty$. For $L^{\infty}(0, T ; X)$ the corresponding condition is \|\|$v(\cdot, t)\left\|_{X}\right\|_{L^{\infty}(0, T)}<\infty$. If $1 \leq p<\infty$ and $X^{*}$ is separable, then $\left(L^{p}(0, T ; X)\right)^{*}=L^{p^{\prime}}\left(0, T ; X^{*}\right)$ with the duality pairing given by

$$
\langle f, v\rangle_{L^{p^{\prime}}\left(0, T ; X^{*}\right)-L^{p}(0, T ; X)}:=\int_{0}^{T}\langle f(\cdot, t), v(\cdot, t)\rangle_{X^{*}-X} d t
$$

(see 28, Corollary 1.3.22]). Furthermore, then $L^{p}(0, T ; X)$ is separable (see [28, Proposition 1.2.29]) and thus every bounded sequence in $L^{p^{\prime}}\left(0, T ; X^{*}\right)$ has a weak* convergent subsequence. We also denote by $C_{w}([0, T) ; X)$ the set of mappings $v:[0, T) \rightarrow X$ defined at every $t \in[0, T)$ and satisfying $t_{j} \rightarrow t$ in $[0, T) \Rightarrow v\left(\cdot, t_{j}\right) \rightarrow$ $v(\cdot, t)$ in $X$.

Whenever $f \in L^{1}(0, T ; X), 0<\delta<T / 2$ and $\theta \in C_{c}^{\infty}(\mathbb{R})$ with $\operatorname{supp}(\theta) \subset(-\delta, \delta)$, we define $f * \theta \in C^{\infty}(\delta, T-\delta ; X)$ by $f * \theta(\cdot, t):=\int_{0}^{T} \theta(t-s) f(\cdot, s) d s \in X$. We record a variant of Young's convolution inequality.

Lemma 2.9. Suppose $p, q, r \in[1, \infty]$ with $1 / p+1 / q=1+1 / r$ and $1 \leq s<\infty$. If $v \in L^{p}\left(0, T ; L^{s}(\Omega)\right)$ and $\theta \in C_{c}^{\infty}(\mathbb{R})$ with $\operatorname{supp}(\theta) \subset(-\delta, \delta)$, then

$$
\|v * \theta\|_{L^{r}\left(\delta, T-\delta ; L^{s}(\Omega)\right)} \leq\|v\|_{L^{p}\left(0, T ; L^{s}(\Omega)\right)}\|\theta\|_{L^{q}(-\delta, \delta)} .
$$

Proof. By Minkowski's integral inequality and Young's convolution inequality,

$$
\begin{aligned}
& \int_{\delta}^{T-\delta}\left(\int_{\Omega}|v * \theta(x, t)|^{s} d x\right)^{\frac{r}{s}} d t \\
= & \int_{\delta}^{T-\delta}\left(\int_{\Omega}\left|\int_{0}^{T} v(x, \tau) \theta(t-\tau) d \tau\right|^{s} d x\right)^{\frac{r}{s}} d t \\
\leq & \int_{\delta}^{T-\delta}\left(\int_{0}^{T}\left(\int_{\Omega}|v(x, \tau)|^{s} d x\right)^{\frac{1}{s}}|\theta(t-\tau)| d \tau\right)^{r} d t \\
= & \int_{\delta}^{T-\delta}\left(\|x \mapsto v(x, \cdot)\|_{L^{s}(\Omega)} *|\theta|(t)\right)^{r} d t \\
\leq & \|v\|_{L^{p}\left(0, T ; L^{s}(\Omega)\right)}^{r}\|\theta\|_{L^{q}(-\delta, \delta)}^{r} .
\end{aligned}
$$


We fix, for the rest of this article, an even mollifier $\chi \in C_{c}^{\infty}(\mathbb{R})$ with $\operatorname{supp}(\chi) \subset$ $(-1,1)$ and $\int_{-1}^{1} \chi(t) d t=1$. We denote $\chi^{\delta}(t):=\delta^{-1} \chi(t / \delta)$ for all $\delta>0$ and $t \in \mathbb{R}$. For every $f \in L^{1}\left(0, T ; L^{1}(\Omega)\right)$ we denote $f_{\delta}:=f * \chi^{\delta}$. For a proof of the following mollifier approximation lemma see [28, Proposition 1.2.32].

Lemma 2.10. Let $0<\epsilon<T / 2$ and suppose $1 \leq p, q<\infty$ and $f \in L^{p}\left(0, T ; L^{q}(\Omega)\right)$. Then $\left\|f_{\delta}-f\right\|_{L^{p}\left(\epsilon, T-\epsilon ; L^{q}(\Omega)\right)} \rightarrow 0$ as $\delta \rightarrow 0$.

The following interpolation inequalities will also be useful to us.

Lemma 2.11. For every $v, w \in L^{\infty}\left(0, T ; L^{2}\left(\Omega, \mathbb{R}^{3}\right)\right) \cap L^{2}\left(0, T ; W^{1,2}\left(\Omega, \mathbb{R}^{3}\right)\right)$ we have

$$
\begin{aligned}
& \|v\|_{L^{4}\left(0, T ; L^{3}(\Omega)\right)} \lesssim \Omega\|v\|_{L^{2}\left(0, T ; W^{1,2}(\Omega)\right.}^{1 / 2}\|v\|_{L^{\infty}\left(0, T ; L^{2}(\Omega)\right)}^{1 / 2}, \\
& \|v \otimes w\|_{L^{1}\left(0, T ; W^{1,3 / 2}(\Omega)\right)} \lesssim \Omega\|v\|_{L^{2}\left(0, T ; W^{1,2}(\Omega)\right)}\|w\|_{L^{2}\left(0, T ; W^{1,2}(\Omega)\right)} \\
& \|v \otimes w\|_{L^{4 / 3}\left(0, T ; L^{2}(\Omega)\right)} \lesssim_{\Omega}\|v\|_{L^{2}\left(0, T ; W^{1,2}(\Omega)\right)}^{3 / 4}\|v\|_{L^{\infty}\left(0, T ; L^{2}(\Omega)\right)}^{1 / 4} \\
& \cdot\|w\|_{L^{2}\left(0, T ; W^{1,2}(\Omega)\right)}^{3 / 4}\|w\|_{L^{\infty}\left(0, T ; L^{2}(\Omega)\right)}^{1 / 4}
\end{aligned}
$$

where $v \otimes w:=\left[v_{i} w_{j}\right]_{i, j=1}^{3}$ is the tensor product of $v$ and $w$.

Proof. The first inequality is a standard interpolation and can be found e.g. at [40, p. 74] (up to a use of the Sobolev embedding $W^{1,2}(\Omega) \subset L^{6}(\Omega)$ ). For the second one note that at a.e. $t \in(0, T)$, Hölder's inequality and the Sobolev embedding $W^{1,2}(\Omega) \hookrightarrow L^{6}(\Omega)$ yield

$$
\||v||\nabla w|\|_{L^{3 / 2}(\Omega)} \leq\|v\|_{L^{6}(\Omega)}\|\nabla w\|_{L^{2}(\Omega)} \lesssim \Omega\|v\|_{W^{1,2}(\Omega)}\|w\|_{W^{1,2}(\Omega)} .
$$

A similar inequality holds for $|w||\nabla w|$ and $|v||w|$, and one then uses the CauchySchwarz inequality on time integrals to finish the proof. Similar reasoning is used to prove the third inequality of the lemma.

We also recall the Aubin-Lions Lemma which we formulate in a form that suffices for the purposes of this article (see [41, Lemma 7.7]).

Lemma 2.12. Let $X, Y$ and $Z$ be reflexive Banach spaces such that $X$ embeds compactly into $Y$ and $Y$ embeds into $Z$. Suppose $1<p<\infty$ and $1 \leq q \leq \infty$. Then $\left\{u \in L^{p}(0, T ; X): \partial_{t} u \in L^{q}(0, T ; Z)\right\}$ embeds compactly into $L^{p}(0, T ; Y)$.

2.4. Leray-Hopf solutions of viscous, resistive MHD equations and the inviscid, non-resistive limit. We recall the definition and present an existence theorem on Leray-Hopf solutions of viscous, resistive 3D MHD equations. When $1<p<\infty$, we denote the relevant function spaces by

$$
\begin{aligned}
& C_{c, \sigma}^{\infty}\left(\Omega, \mathbb{R}^{3}\right):=\left\{\varphi \in C_{c}^{\infty}\left(\Omega, \mathbb{R}^{3}\right): \operatorname{div} \varphi=0\right\}, \\
& L_{\sigma}^{p}\left(\Omega, \mathbb{R}^{3}\right):=\overline{C_{c, \sigma}^{\infty}\left(\Omega, \mathbb{R}^{3}\right)}{ }^{L^{p}\left(\Omega, \mathbb{R}^{3}\right)}=\left\{v \in L^{p}\left(\Omega, \mathbb{R}^{3}\right): \operatorname{div} v=0,\left.v \cdot n\right|_{\Gamma}=0\right\}, \\
& W_{0, \sigma}^{1, p}\left(\Omega, \mathbb{R}^{3}\right):=\overline{C_{c, \sigma}^{\infty}\left(\Omega, \mathbb{R}^{3}\right)} W_{0}^{1, p}\left(\Omega, \mathbb{R}^{3}\right)=\left\{v \in W_{0}^{1, p}\left(\Omega, \mathbb{R}^{3}\right): \operatorname{div} v=0\right\}, \\
& W_{\sigma}^{1, p}\left(\Omega, \mathbb{R}^{3}\right):=\left\{v \in W^{1, p}\left(\Omega, \mathbb{R}^{3}\right): \operatorname{div} v=0,\left.v \cdot n\right|_{\Gamma}=0\right\} .
\end{aligned}
$$

(for the two identities see e.g. [25, Theorems III.2.3 and III.4.1]). Leray-Hopf solutions of MHD are defined by the following standard variational formulation. 
Definition 2.13. Let $u_{0}, b_{0} \in L_{\sigma}^{2}\left(\Omega, \mathbb{R}^{3}\right)$. Suppose that $u \in C_{w}\left([0, T) ; L_{\sigma}^{2}\left(\Omega, \mathbb{R}^{3}\right)\right) \cap$ $L^{2}\left(0, T ; W_{0, \sigma}^{1,2}(\Omega)\right)$ and $b \in C_{w}\left([0, T) ; L_{\sigma}^{2}\left(\Omega, \mathbb{R}^{3}\right)\right) \cap L^{2}\left(0, T ; W_{\sigma}^{1,2}(\Omega)\right)$ satisfy $\partial_{t} u \in$ $L^{1}\left(0, T ;\left(W_{0, \sigma}^{1,2}\left(\Omega, \mathbb{R}^{3}\right)\right)^{*}\right)$ and $\partial_{t} b \in L^{1}\left(0, T ;\left(W_{\sigma}^{1,2}\left(\Omega, \mathbb{R}^{3}\right)\right)^{*}\right)$, and that

$$
\begin{aligned}
& \left\langle\partial_{t} u, \varphi\right\rangle+\int_{\Omega}(u \cdot \nabla u-b \cdot \nabla b) \cdot \varphi+\nu \int_{\Omega} \nabla u: \nabla \varphi=0, \\
& \left\langle\partial_{t} b, \theta\right\rangle+\int_{\Omega} b \times u \cdot \operatorname{curl} \theta+\mu \int_{\Omega} \operatorname{curl} b \cdot \operatorname{curl} \theta=0
\end{aligned}
$$

hold at a.e. $t \in[0, T)$ and every $\varphi \in W_{0, \sigma}^{1,2}\left(\Omega, \mathbb{R}^{3}\right)$ and $\theta \in W_{\sigma}^{1,2}\left(\Omega, \mathbb{R}^{3}\right)$. Suppose furthermore that $u(\cdot, 0)=u_{0}$ and $b(\cdot, 0)=b_{0}$ and that $u$ and $b$ satisfy the energy inequality

$$
\begin{aligned}
& \frac{1}{2} \int_{\Omega}\left(|u(x, t)|^{2}+|b(x, t)|^{2}\right) d x \\
+ & \int_{0}^{t} \int_{\Omega}\left(\nu|\nabla u(x, \tau)|^{2}+\mu|\operatorname{curl} b(x, \tau)|^{2}\right) d x d \tau \\
\leq & \frac{1}{2} \int_{\Omega}\left(\left|u_{0}(x)\right|^{2}+\left|b_{0}(x)\right|^{2}\right) d x
\end{aligned}
$$

for all $t \in(0, T)$. Then $(u, b)$ is called a Leray-Hopf solution of (1.1)-(1.6).

Note that 2.4 captures in a weak sense the condition $(\operatorname{curl} b) \times\left. n\right|_{\Gamma}=0$. Also note that 2.4 and the condition $b(\cdot, 0)=b_{0}$ imply

$$
\int_{0}^{T} \partial_{t} \eta \int_{\Omega} b \cdot \theta-\int_{0}^{T} \eta \int_{\Omega} b \times u \cdot \operatorname{curl} \theta-\mu \int_{0}^{t} \eta \int_{\Omega} \operatorname{curl} b \cdot \operatorname{curl} \theta+\eta(0) \int_{\Omega} b_{0} \cdot \theta=0
$$

for all $\eta \in C_{c}^{\infty}([0, T))$ and $\theta \in W_{\sigma}^{1,2}\left(\Omega, \mathbb{R}^{3}\right)$. As mentioned in the introduction, we present a proof of the following theorem in the Appendix.

Theorem 2.14. Let $u_{0}, b_{0} \in L_{\sigma}^{2}\left(\Omega ; \mathbb{R}^{3}\right)$. Then there exists a Leray-Hopf solution $(u, b)$ of 1.1$)-(1.6)$.

Theorems 1.2 and 1.5 do not assume that the inviscid, non-resistive (i.e. ideal) limit, defined below, holds. However, we mention the notion for completeness and also because it falls under the scope of Corollary 1.3 . It is a fundamental open problem under what conditions the inviscid, non-resistive limit holds in 3D MHD (see [14, 48, 49], 50], [51 and [53] for partial results).

Definition 2.15. Suppose viscosities $\nu_{j}>0$ and resistivities $\mu_{j}>0$ satisfy $\nu_{j}, \mu_{j} \rightarrow 0$ and that divergence-free initial datas $u_{j, 0} \rightarrow u_{0}$ and $b_{j, 0} \rightarrow b_{0}$ in $L_{\sigma}^{2}\left(\Omega, \mathbb{R}^{3}\right)$. Assume that for every $j \in \mathbb{N},\left(u_{j}, b_{j}\right)$ is a Leray-Hopf solution of 11.1 (1.6) and that $u, b \in L^{\infty}\left(0, T ; L_{\sigma}^{2}\left(\Omega, \mathbb{R}^{3}\right)\right)$ form a solution of 1.1 -1.4 with $\mu=$ $\nu=0$. We say that $(u, b)$ is the inviscid, non-resistive limit or ideal limit of $\left(u_{j}, b_{j}\right)$ (in the energy norm) if $\left\|u_{j}-u\right\|_{L^{\infty}\left(0, T ; L^{2}(\Omega)\right)} \rightarrow 0$ and $\left\|b_{j}-b\right\|_{L^{\infty}\left(0, T ; L^{2}(\Omega)\right)} \rightarrow 0$. We then also say that the inviscid, non-resistive limit holds for $\left(u_{j}, b_{j}\right)$ and $(u, b)$.

\section{Vector potentials and gauge Dependence of magnetic helicity}

The aim of this section is to discuss the notion of magnetic helicity in multiply connected domains and to recall the existence of vector potentials satisfying the assumptions of Corollary 1.6 . 
3.1. Magnetic helicity in multiply connected domains. We first recall the Helmholtz-Weyl decomposition of $L^{2}\left(\Omega, \mathbb{R}^{3}\right)$ which is, in fact, valid in every domain of $\mathbb{R}^{n}$ for all $n \geq 2$ (see [24, Theorem III.1.1]).

Theorem 3.1. $L^{2}(\Omega)=L_{\sigma}^{2}\left(\Omega, \mathbb{R}^{3}\right) \oplus \nabla W^{1,2}\left(\Omega, \mathbb{R}^{3}\right)$.

In [23, Foias and Temam performed a further direct decomposition of $L_{\sigma}^{2}\left(\Omega, \mathbb{R}^{3}\right)$ into a part that has a vanishing flux across the cuts and an harmonic part (see [23, Proposition 1.1] or [47, Appendix I, Lemma 1.4]). We present the decomposition of Foias and Temam in the notation of 52 .

Theorem 3.2. $L_{\sigma}^{2}\left(\Omega, \mathbb{R}^{3}\right)=L_{\Sigma}^{2}\left(\Omega, \mathbb{R}^{3}\right) \oplus L_{H}^{2}\left(\Omega, \mathbb{R}^{3}\right)$, where

$$
\begin{aligned}
& L_{\Sigma}^{2}\left(\Omega, \mathbb{R}^{3}\right):=\left\{v \in L_{\sigma}^{2}\left(\Omega, \mathbb{R}^{3}\right): \int_{\Sigma_{i}} v(x) \cdot n(x) d S(x)=0 \text { for } i=1, \ldots, N\right\}, \\
& L_{H}^{2}\left(\Omega, \mathbb{R}^{3}\right):=\left\{v \in L_{\sigma}^{2}\left(\Omega, \mathbb{R}^{3}\right): \operatorname{curl} v=0\right\} .
\end{aligned}
$$

By Theorem 2.8, $L_{H}^{2}\left(\Omega, \mathbb{R}^{3}\right) \subset W_{\sigma}^{1,2}\left(\Omega, \mathbb{R}^{3}\right)$.

Definition 3.3. We denote the projections onto $L_{\Sigma}^{2}\left(\Omega, \mathbb{R}^{3}\right)$ and $L_{H}^{2}\left(\Omega, \mathbb{R}^{3}\right)$ by $P_{\Sigma}: L_{\sigma}^{2}\left(\Omega, \mathbb{R}^{3}\right) \rightarrow L_{\Sigma}^{2}\left(\Omega, \mathbb{R}^{3}\right)$ and $P_{H}: L_{\sigma}^{2}\left(\Omega, \mathbb{R}^{3}\right) \rightarrow L_{H}^{2}\left(\Omega, \mathbb{R}^{3}\right)$. For every $v \in$ $L_{\sigma}^{2}\left(\Omega, \mathbb{R}^{3}\right)$ we denote $v_{\Sigma}:=P_{\Sigma} v$ and $v_{H}:=P_{H} v$.

The vector space $L_{H}^{2}\left(\Omega, \mathbb{R}^{3}\right)$ is $N$-dimensional. For a characterisation of an orthonormal basis $\left\{h_{1}, \ldots, h_{N}\right\}$ of $L_{H}^{2}\left(\Omega, \mathbb{R}^{3}\right)$ see [47, Appendix I, Lemma 1.3] or [1, Proposition 3.14]. Theorems 3.1 and 3.2 yield the decomposition

$$
L^{2}\left(\Omega, \mathbb{R}^{3}\right)=L_{\Sigma}^{2}\left(\Omega, \mathbb{R}^{3}\right) \oplus \operatorname{ker}(\operatorname{curl}) .
$$

Furthermore, $L_{\sigma}^{2}\left(\Omega, \mathbb{R}^{3}\right) \subset\left\{\operatorname{curl} \psi: \psi \in W^{1,2}\left(\Omega, \mathbb{R}^{3}\right)\right\}$ (see [47, Appendix I, Proposition 1.3]). We record the following simple observation.

Proposition 3.4. Suppose $b \in L_{\sigma}^{2}\left(\Omega, \mathbb{R}^{3}\right)$. Then the value $\int_{\Omega} \psi(x) \cdot b(x) d x$ is independent of the solution $\psi \in W^{1,2}\left(\Omega, \mathbb{R}^{3}\right)$ of $\operatorname{curl} \psi=b$ if and only if $b \in$ $L_{\Sigma}^{2}\left(\Omega, \mathbb{R}^{3}\right)$.

Proof. If $b \in L_{\sigma}^{2}\left(\Omega, \mathbb{R}^{3}\right)$ and $\int_{\Omega} \phi(x) \cdot b(x) d x=0$ for all $\phi \in W^{1,2}\left(\Omega, \mathbb{R}^{3}\right)$ with $\operatorname{curl} \phi=0$, then in particular $\int_{\Omega} b(x) \cdot h_{i}(x) d x=0$ for all $i \in\{1, \ldots, N\}$, giving $b \in L_{\Sigma}^{2}\left(\Omega, \mathbb{R}^{3}\right)$. The converse follows immediately from 3.1.

Consequently, magnetic helicity is independent of the vector potential for every $b(\cdot, t) \in L_{\sigma}^{2}\left(\Omega, \mathbb{R}^{3}\right)$ precisely when $L_{H}^{2}\left(\Omega, \mathbb{R}^{3}\right)=\{0\}$. In Proposition 3.5 this helps us to characterise, in multiply connected domains, those magnetic fields whose magnetic helicity is conserved for every vector potential.

Proposition 3.5. Suppose the mappings $u_{j}, b_{j}, u_{j, 0}, b_{j, 0}, u, b, u_{0}, b_{0}$ satisfy the assumptions of Theorem 1.5. Then the following conditions are equivalent.

(i) $\int_{\Omega} \psi(x, t) \cdot b(x, t) d x$ is a.e. constant for every $\psi \in L^{\infty}\left(0, T ; W^{1,2}\left(\Omega, \mathbb{R}^{3}\right)\right)$ with $\operatorname{curl} \psi=b$.

(ii) $b_{H}=0$.

(iii) $b_{0, H}=0$.

If $\Omega$ is multiply connected, there exist $u_{j}, b_{j}, u_{j, 0}, b_{j, 0}, u, b, u_{0}, b_{0}$ such that (i)-(iii) are not satisfied. 
Proof. The equivalence (i) $\Leftrightarrow$ (ii) is a direct corollary of Theorem 1.5 and Proposition 3.4 and the equivalence (ii) $\Leftrightarrow$ (iii) follows immediately from Lemma 4.3 . The last claim follows by combining Lemma 4.3 and Theorem 2.14 .

Proposition 3.5 indicates that in multiply connected domains, magnetic helicity conservation can only hold in the weak ideal limit if some restrictions are imposed on the vector potential.

3.2. Good vector potentials. As stated in Corollary 1.6, a condition that allows magnetic helicity conservation in multiply connected domains is given by

$$
\psi^{\Sigma} \times\left. n\right|_{\Gamma}=0 \quad \text { and } \quad \psi_{0}^{\Sigma} \times\left. n\right|_{\Gamma}=0 .
$$

We will, in fact, obtain Theorem 1.5 as a consequence of the fact that $(3.2)$ leads to magnetic helicity conservation. For more information on condition 3.2 see e.g. [1], 32] and [52.

Our next aim is to specify vector potentials that satisfy $(3.2)$. For the $L_{\Sigma}^{2}$ part of the magnetic field we essentially use vector potentials found by Borchers and Sohr in [4, Corollary 2.2]. The boundary condition $\left\langle\partial_{n}(\operatorname{div} \psi), 1\right\rangle_{\Gamma_{i}}=0$, added by Amrouche, Bernardi, Dauge and Girault in [1, ensures uniqueness. Theorem 3.6 follows from [1, Corollary 3.19] and [1, Theorem 3.20].

Theorem 3.6. For every $v \in L_{\Sigma}^{2}\left(\Omega, \mathbb{R}^{3}\right)$ there exists a unique $T_{\Sigma} v:=\Psi^{\Sigma} \in$ $W_{0}^{1,2}\left(\Omega, \mathbb{R}^{3}\right)$ such that

$$
\operatorname{curl} \Psi^{\Sigma}=v, \quad \operatorname{div}\left(\Delta \Psi^{\Sigma}\right)=0, \quad\left\langle\partial_{n}\left(\operatorname{div} \Psi^{\Sigma}\right), 1\right\rangle_{\Gamma_{i}}=0 \quad(i=1, \ldots, K) .
$$

Furthermore, $T_{\Sigma}: L_{\Sigma}^{2}\left(\Omega, \mathbb{R}^{3}\right) \rightarrow W_{0}^{1,2}\left(\Omega, \mathbb{R}^{3}\right)$ is linear and bounded.

For the space $L_{H}^{2}\left(\Omega, \mathbb{R}^{3}\right)$ a natural choice of potentials is a special case of [1, Theorem 3.12] and [1, Corollary 3.16]:

Theorem 3.7. For every $v \in L_{H}^{2}\left(\Omega, \mathbb{R}^{3}\right)$ there exists a unique $T_{H} v:=\psi^{H} \in$ $W^{1,2}\left(\Omega, \mathbb{R}^{3}\right) \cap L_{\Sigma}^{2}\left(\Omega, \mathbb{R}^{3}\right)$ such that $\operatorname{curl}\left(\psi^{H}\right)=v$. Furthermore, $T_{H}: L_{H}^{2}\left(\Omega, \mathbb{R}^{3}\right) \rightarrow$ $W^{1,2}\left(\Omega, \mathbb{R}^{3}\right)$ is linear and bounded.

We use Theorems 3.6 and 3.7 to record an existence theorem about vector potentials satisfying $(3.2)$.

Corollary 3.8. For every $v \in L^{\infty}\left(0, T ; L_{\sigma}^{2}\left(\Omega, \mathbb{R}^{3}\right)\right)$, the mappings $\Psi^{\Sigma}(x, t):=$ $T_{\Sigma} v_{\Sigma}(x, t)$ and $\psi^{H}(x, t):=T_{H} v_{H}(x, t)$ belong to $L^{\infty}\left(0, T ; W^{1,2}\left(\Omega, \mathbb{R}^{3}\right)\right)$ and satisfy $\operatorname{curl} \Psi^{\Sigma}=v_{\Sigma}, \operatorname{curl} \psi^{H}=v_{H}$ and 3.2 .

Given $v \in L^{\infty}\left(0, T ; L_{\sigma}^{2}\left(\Omega, \mathbb{R}^{3}\right)\right)$, the time-dependent mappings $T_{\Sigma} v_{\Sigma}$ and $T_{H} v_{H}$ are strongly measurable, which follows from the fact that $T_{\Sigma} \circ P_{\Sigma}$ and $T_{H} \circ P_{H}$ are bounded linear operators from $L_{\sigma}^{2}\left(\Omega, \mathbb{R}^{3}\right)$ into $W^{1,2}\left(\Omega, \mathbb{R}^{3}\right)$.

Remark 3.9. Another choice of vector potentials that satisfies (3.2) (a special case of the Coulomb gauge) is given in [1, Theorem 3.17] and [1, Corollary 3.19]: for every $v \in L_{\Sigma}^{2}\left(\Omega, \mathbb{R}^{3}\right)$ there exists a unique $\phi \in W^{1,2}\left(\Omega, \mathbb{R}^{3}\right)$ with $\operatorname{curl} \phi=v$, $\operatorname{div} \phi=0, \phi \times n=0$ on $\Gamma$ and $\langle\phi \cdot n, 1\rangle_{\Gamma_{i}}=0$ for all $i \in\{1, \ldots, N\}$ - the condition $\left.\psi^{\Sigma} \cdot n\right|_{\Gamma}=0$ is thus traded for $\operatorname{div} \phi=0$.

We will also need a scalar potential for time-dependent curl-free $L^{p}$ vector fields in simply connected domains. 
Lemma 3.10. Let $1 \leq p \leq \infty$ and $1<q<\infty$, and suppose $\Omega^{\prime} \subset \Omega$ is a simply connected domain with smooth boundary. If $v \in L^{p}\left(0, T ; L^{q}\left(\Omega^{\prime}, \mathbb{R}^{3}\right)\right)$ is curl-free, then there exists a unique $g \in L^{p}\left(0, T ; W^{1, q}\left(\Omega^{\prime}\right)\right)$ such that $v=\nabla g$ and $\int_{\Omega^{\prime}} g(x, t) d x=0$. Furthermore, $\|g(\cdot, t)\|_{W^{1, q}\left(\Omega^{\prime}\right)} \Omega_{\Omega^{\prime}, q}\|v(\cdot, t)\|_{L^{q}\left(\Omega^{\prime}\right)}$ for a.e. $t \in(0, T)$.

Proof. For the existence of $g(\cdot, t)$ a.e. $t \in(0, T)$ see e.g. [25, Lemma III.1.1]. Given $t \in(0, T)$ the uniqueness of $g(\cdot, t)$ is clear and the norm estimate follows from the Poincaré inequality. Since $v(\cdot, t) \mapsto g(\cdot, t)$ is linear, $g$ is strongly measurable.

\section{The Proof of Theorems 1.2 And 1.5}

Theorem 1.5 is proved in this section, and Theorem 1.2 is obtained as a special case. We begin by motivating our decomposition of vector potentials.

4.1. The decomposition of vector potentials. Given a weak ideal or nonresistive limit $(u, b)$, our aim is to compute $\int_{\Omega} \psi(x, t) \cdot b(x, t) d x$ at a.e. $t \in(0, T)$ for every vector potential $\psi \in L^{\infty}\left(0, T ; W^{1,2}\left(\Omega, \mathbb{R}^{3}\right)\right)$ of $b$. However, we do not assume that $(u, b)$ satisfies the ideal MHD equations and so no neat formula for $\int_{\Omega} \psi(x, t)$. $b(x, t) d x$ is readily available. We therefore wish to relate $\int_{\Omega} \psi(x, t) \cdot b(x, t) d x$ to $\int_{\Omega} \psi_{j}(x, t) \cdot b_{j}(x, t) d x$ and compute the latter for every $\psi_{j}$.

A natural idea for computing $\int_{\Omega} \psi_{j}(x, t) \cdot b_{j}(x, t) d x$ (which works without major complications in simply connected domains) is to write

$$
\begin{aligned}
\int_{\Omega} \psi_{j}(x, t) \cdot b_{j}(x, t) d x & =\int_{0}^{t} \int_{\Omega}\left[\partial_{\tau} \psi_{j}(x, \tau) \cdot b_{j}(x, \tau)+\psi_{j}(x, \tau) \cdot \partial_{\tau} b_{j}(x, \tau)\right] d x d \tau \\
& +\int_{\Omega} \psi_{j}(x, 0) \cdot b_{j}(x, 0) d x
\end{aligned}
$$

and use the induction equation

$$
\partial_{t} b_{j}+\operatorname{curl}\left(b_{j} \times u_{j}\right)+\mu_{j} \operatorname{curl} \operatorname{curl} b_{j}=0
$$

on $\partial_{\tau} \psi_{j}$ and $\partial_{\tau} b_{j}$. In the multiply connected case, however, 4.1) leads (formally) to $\partial_{t} \psi_{j}=-b_{j} \times u_{j}-\mu_{j} \operatorname{curl} b_{j}+\sum_{i=1}^{N} d_{i}(t) h_{i}+\nabla g$, where $\left\{h_{1}, \ldots, h_{N}\right\}$ is an orthonormal basis of $L_{H}^{2}\left(\Omega, \mathbb{R}^{3}\right)$, and the product $\sum_{i=1}^{N} d_{i}(t) h_{i} \cdot b_{j}$ seems very difficult to control. We therefore maneuver carefully in the proof of Lemma 4.5 to make sure that we do not multiply $\partial_{t} \psi_{j}$ and $b_{j, H}$ at any point of the argument.

The considerations above prompt us to decompose $\psi_{j}$ and take advantage of the differences between $b_{j, \Sigma}$ and $b_{j, H}$. Using the notation of Corollary 3.8 , we write

$$
\psi_{j}=\left(\psi_{j}-\Psi_{j}^{\Sigma}-\psi_{j}^{H}\right)+\Psi_{j}^{\Sigma}+\psi_{j}^{H} .
$$

In 4.2 ,,$\partial_{t} \psi_{j}^{H}=0$, while the condition $\Psi_{j}^{\Sigma} \times\left. n\right|_{\Gamma}=0$ ensures that many natural integrations by parts do not create unwanted boundary terms. These properties play a key role in the proof of Lemma 4.5 . Finally, the 'bad part' $\psi_{j}-\psi_{j}^{\Sigma}-\psi_{j}^{H}$ is curl-free and, consequently, $\int_{\Omega}\left(\psi_{j}(x, t)-\Psi_{j}^{\Sigma}(x, t)-\psi_{j}^{H}(x)\right) \cdot b_{j}(x, t) d x$ can be given a simple formula (see Lemma 4.2).

Furthermore, while $\psi_{j}$ need not converge to $\psi$ in any useful sense, the 'good parts' $\Psi_{j}^{\Sigma}+\psi_{j}^{H}$ of the potentials satisfy

$$
\Psi_{j}^{\Sigma}+\psi_{j}^{H} \rightarrow \Psi^{\Sigma}+\psi^{H} \quad \text { in } L_{l o c}^{2}\left(0, T ; L^{2}\left(\Omega, \mathbb{R}^{3}\right)\right)
$$


(see Lemma 4.4. The basic reason is that $\Psi_{j}^{\Sigma}$ and $\psi_{j}^{H}$ depend linearly on $b_{j}$, which allows us to exploit the weak-* convergence $b_{j} \stackrel{*}{\rightarrow} b$ in $L^{\infty}\left(0, T ; L_{\sigma}^{2}\left(\Omega, \mathbb{R}^{3}\right)\right)$.

4.2. An overview of the proof. The proof of Theorem 1.5 is reduced to the special case of $\psi_{j}=\Psi_{j}^{\Sigma}+\psi_{j}^{H}$ and $\psi=\Psi^{\Sigma}+\psi^{H}$ in Lemma 4.2 . We therefore introduce a shorthand notation for magnetic helicity in this gauge.

Definition 4.1. Given $v \in L^{\infty}\left(0, T ; L_{\sigma}^{2}\left(\Omega, \mathbb{R}^{3}\right)\right)$ and $\Psi^{\Sigma}:=T_{\Sigma} v, \psi^{H}:=T_{H} v$ we denote

$$
\mathscr{M}(v ; t):=\int_{\Omega}\left(\Psi^{\Sigma}(x, t)+\psi^{H}(x, t)\right) \cdot v(x, t) d x .
$$

Our aim is to show that

$$
\mathscr{M}\left(b_{j} ; t\right)=\mathscr{M}\left(b_{j} ; 0\right)-2 \mu_{j} \int_{0}^{t} \int_{\Omega} b_{j}(x, \tau) \cdot \operatorname{curl} b_{j}(x, \tau) d x d \tau
$$

for every $j \in \mathbb{N}$ and $t \in[0, T)$ and that given $\eta \in C_{c}^{\infty}(0, T)$,

$$
\int_{0}^{T} \eta(t) \mathscr{M}(b ; t) d t=\lim _{j \rightarrow \infty} \int_{0}^{T} \eta(t) \mathscr{M}\left(b_{j} ; t\right) d t=\lim _{j \rightarrow \infty} \int_{0}^{T} \eta(t) \mathscr{M}\left(b_{j} ; 0\right) d t .
$$

Once (4.5) is proved, (1.11) follows for $\psi=\Psi^{\Sigma}+\psi^{H}$ rather easily (see Lemma 4.7).

The leftmost equality in 4.5 is proved by showing 4.3 and recalling that $b_{j} \rightarrow b$ in $L^{2}\left(0, T ; L^{2}\left(\Omega, \mathbb{R}^{3}\right)\right)$. The proof of $(4.3)$ uses the Aubin-Lions Lemma as a main tool and is presented in $\$ 4.5$. The rightmost equality of 4.5 is proved in $\$ 4.6$ by showing that the double integral on the right-hand side of (4.4) vanishes at the limit $j \rightarrow \infty$.

We finally mention that in the proof of Theorem 1.5 we will on several occasions pass to a subsequence without relabeling it. The limit (1.11) will however hold for the whole sequence $\left(b_{j}\right)_{j=1}^{\infty}$ as every subsequence will have a subsequence satisfying (1.11).

4.3. Reduction to good vector potentials. The following lemma shows that it suffices to prove the claims of Theorem 1.5 and Corollary 1.6 for the potentials of Definition 4.1. It also indicates to what extent gauge invariance of magnetic helicity fails in multiply connected domains.

Lemma 4.2. Suppose $\psi=\psi^{\Sigma}+\psi^{H} \in L^{\infty}\left(0, T ; W^{1,2}\left(\Omega, \mathbb{R}^{3}\right)\right)$ is a vector potential of $v \in L^{\infty}\left(0, T ; L_{\sigma}^{2}\left(\Omega, \mathbb{R}^{3}\right)\right)$. Then

$$
\int_{\Omega} \psi(x, t) \cdot v(x, t) d x=\mathscr{M}(v ; t)-\int_{\Gamma} \psi^{\Sigma}(x, t) \times n \cdot \psi^{H}(x) d x
$$

at a.e. $t \in(0, T)$.

Proof. By using the definition of $\mathscr{M}(v ; t)$ and the facts that $\psi^{\Sigma}-\Psi^{\Sigma} \in \operatorname{ker}$ curl $=$ $L_{\Sigma}^{2}\left(\Omega, \mathbb{R}^{3}\right)^{\perp}$ and $\Psi^{\Sigma} \times\left. n\right|_{\Gamma}=0$ we obtain

$$
\begin{aligned}
\int_{\Omega} \psi(x, t) \cdot v(x, t) d x & =\mathscr{M}(v ; t)+\int_{\Omega}\left(\psi^{\Sigma}(x, t)-\Psi^{\Sigma}(x, t)\right) \cdot v_{H}(x, t) d S(x) \\
& =\mathscr{M}(v ; t)+\int_{\Gamma} \psi^{H}(x) \times n \cdot\left(\psi^{\Sigma}(x, t)-\Psi^{\Sigma}\right) d S(x) \\
& =\mathscr{M}(v ; t)-\int_{\Gamma} \psi^{\Sigma}(x, t) \times n \cdot \psi^{H}(x) d x
\end{aligned}
$$

at a.e. $t \in(0, T)$. 
4.4. Stationarity of the harmonic parts of magnetic fields. We next show that for Leray-Hopf solutions and their weak ideal limits, the harmonic part of the magnetic field is stationary.

Lemma 4.3. Under the assumptions of Theorem 1.5, for every $j \in \mathbb{N}$ the harmonic parts $b_{j, H}$ and $b_{H}$ are of the forms $b_{j, H}(x, t)=b_{j, 0, H}(x)$ and $b_{H}(x, t)=b_{0, H}(x)=$ $\lim _{j \rightarrow \infty} b_{j, 0, H}(x)$.

Proof. Given $j \in \mathbb{N}$ we write $b_{j, H}(x, t)=\sum_{i=1}^{N} c_{i}^{j}(t) h_{i}(x)$ and set out to prove that $c_{j}^{1}, \ldots, c_{j}^{N}$ are constants independent of $t$. We fix $i \in\{1, \ldots, N\}$ and first show that $c_{j}^{i}$ is continuous. Since $b_{j}$ is weakly $L^{2}$-continuous in time, we get

$$
c_{i}^{j}\left(t_{k}\right)=\int_{\Omega} b_{j}\left(x, t_{k}\right) \cdot h_{i}(x) d x \rightarrow \int_{\Omega} b_{j}(x, t) \cdot h_{i}(x) d x=c_{i}^{j}(t)
$$

whenever $t_{k} \rightarrow t$ in $[0, T)$. Now let $\eta \in C_{c}^{\infty}(0, T)$ and set $\theta(x, t):=\eta(t) h_{i}(x)$ in 2.5, getting $\int_{0}^{T} \eta^{\prime}(t) c_{i}^{j}(t) d t=0$, which implies that $c_{i}^{j}(t)=c_{i}^{j}(0)$ for all $t \in[0, T)$. Fixing $i \in\{1, \ldots, N\}$ and $\eta \in C_{c}^{\infty}(0, T)$ we get

$c_{i}^{j}(0) \int_{0}^{T} \eta(t) d t=\int_{0}^{T} \int_{\Omega} \eta(t) h_{i}(x) \cdot b_{j}(x, t) d x d t \rightarrow \int_{0}^{T} \int_{\Omega} \eta(t) h_{i}(x) \cdot b(x, t) d x d t$,

which yields the statement on $b_{H}$.

4.5. Strong convergence of good vector potentials. The aim of this section is to prove (4.3) via the Aubin-Lions Lemma. This requires uniform control of the norms $\left\|\partial_{t} \psi_{j}\right\|_{L^{1}(0, T ; X)}$ in some (reflexive) Banach space $X \supset L^{2}\left(\Omega, \mathbb{R}^{3}\right)$. Note that since $\partial_{t} \psi_{j}^{H}=0,1.2$ yields

$$
\operatorname{curl}\left(\partial_{t} \psi_{j}^{\Sigma}+b_{j} \times u_{j}+\mu_{j} \operatorname{curl} b_{j}\right)=0 .
$$

If $\Omega^{\prime} \subset \Omega$ is a simply connected subdomain, we can thus write $\partial_{t} \psi_{j}^{\Sigma}+b_{j} \times u_{j}+$ $\mu_{j} \operatorname{curl} b_{j}=\nabla g$ in $\Omega^{\prime}$. It is, however, not immediately clear how well-behaved $\partial_{t} \psi_{j}^{\Sigma}$ (and, thus, $g$ ) is. In order to circumvent this issue we mollify in time via the functions $t \mapsto \chi^{\delta}(t)$ mentioned in $\$ 2.3$ and write $\partial_{t}\left(\psi_{j}^{\Sigma} * \chi^{\delta}\right)=\psi_{j}^{\Sigma} * \partial_{t} \chi^{\delta}$.

Lemma 4.4. The vector potentials $\Psi_{j}^{\Sigma}+\psi_{j}^{H}$ converge in $L_{\text {loc }}^{2}\left(0, T ; L^{2}\left(\Omega, \mathbb{R}^{3}\right)\right)$ to the vector potential $\Psi^{\Sigma}+\psi^{H}$ of b.

Proof. Lemma 4.3 implies that $\psi_{j}^{H}$ converges to $\psi^{H}$ in $L^{2}\left(0, T ; L^{2}\left(\Omega, \mathbb{R}^{3}\right)\right)$. The more elaborate part is the strong convergence of the potentials $\Psi_{j}^{\Sigma}$.

We fix a sequence of numbers $\epsilon_{j} \in(0, T / 2)$. We then choose another sequence of numbers $\delta_{j} \in\left(0, \epsilon_{j}\right)$ such that $\left\|\Psi_{j}^{\Sigma} * \chi^{\delta_{j}}-\Psi_{j}^{\Sigma}\right\|_{L^{2}\left(\epsilon_{j}, T-\epsilon_{j}\right)}<1 / j$ for all $j \in$ $\mathbb{N}$, so that it suffices to prove the convergence of the sequence $\left(\Psi_{j}^{\Sigma} * \chi^{\delta_{j}}\right)_{j=1}^{\infty}$ in $L_{l o c}^{2}\left(0, T ; ; L^{2}\left(\Omega, \mathbb{R}^{3}\right)\right)$. We fix a non-empty simply connected, smooth subdomain $\Omega^{\prime} \subset \Omega$ and aim to show that

$$
\sup _{j \in \mathbb{N}}\left(\left\|\Psi_{j}^{\Sigma} * \chi^{\delta_{j}}\right\|_{L^{2}\left(\epsilon, T-\epsilon ; W^{1,2}\left(\Omega, \mathbb{R}^{3}\right)\right)}+\left\|\partial_{t} \Psi_{j}^{\Sigma} * \chi^{\delta_{j}}\right\|_{L^{2}\left(\epsilon, T-\epsilon ;\left(W_{0, \sigma}^{1,4}\left(\Omega^{\prime}, \mathbb{R}^{3}\right)\right)^{*}\right)}\right)<\infty ;
$$

the Aubin-Lions Lemma then gives norm convergence of a subsequence of $\left(\Psi_{j}^{\Sigma}\right)_{j=1}^{\infty}$ to some $\tilde{\psi} \in L_{l o c}^{2}\left(0, T ; L^{2}(\Omega)\right)$. On the other hand, for a further subsequence, $\Psi_{j}^{\Sigma}=T_{\Sigma} b_{j, \Sigma} \rightarrow T_{\Sigma} b_{\Sigma}$ in $L^{2}\left(0, T ; W^{1,2}\left(\Omega, \mathbb{R}^{3}\right)\right)$, and thus $\tilde{\psi}=T_{\Sigma} b_{\Sigma}=: \Psi^{\Sigma}$. 
For (4.7) we fix $j \in \mathbb{N}$ and use the fact that by Lemma 3.6. Theorem 2.14 and Lemma 2.9

$$
\begin{aligned}
\left\|\Psi_{j}^{\Sigma} * \chi^{\delta_{j}}\right\|_{L^{2}\left(\epsilon, T-\epsilon ; W^{1,2}\left(\Omega, \mathbb{R}^{3}\right)\right)} & \lesssim\left\|b_{j, \Sigma} * \chi^{\delta_{j}}\right\|_{L^{2}\left(\epsilon, T-\epsilon ; L^{2}(\Omega)\right)} \leq\left\|b_{j, \Sigma}\right\|_{L^{2}\left(0, T ; L^{2}(\Omega)\right)} \\
& \leq\left\|b_{j}\right\|_{L^{2}\left(0, T ; L^{2}(\Omega)\right)} \leq \sqrt{T}\left\|b_{j, 0}\right\|_{L^{2}(\Omega)} .
\end{aligned}
$$

In order to control the norm of $\partial_{t}\left(\Psi_{j}^{\Sigma} * \chi^{\delta_{j}}\right)$ we note that $\partial_{t}\left(\Psi_{j} * \chi^{\delta_{j}}\right)=\Psi_{j}^{\Sigma} * \partial_{t} \chi^{\delta_{j}} \in$ $L^{\infty}\left(\epsilon, T-\epsilon ; W_{0}^{1,2}\left(\Omega^{\prime}\right)\right)$, and so Lemma 3.10 and 4.6 yield

$$
\Psi_{j}^{\Sigma} * \partial_{t} \chi^{\delta_{j}}+\left(b_{j} \times u_{j}\right) * \chi^{\delta_{j}}-\mu_{j} \operatorname{curl} b_{j} * \chi^{\delta_{j}}=\nabla g_{j},
$$

where $g_{j} \in L^{\infty}\left(\epsilon, T-\epsilon ; W^{1,2}\left(\Omega^{\prime}\right)\right)$. We estimate, at every $t \in(\epsilon, T-\epsilon)$,

$$
\begin{aligned}
\left|\int_{\Omega^{\prime}} \partial_{t}\left[\Psi_{j}^{\Sigma} * \chi^{\delta_{j}}\right](x, t) \cdot v(x) d x\right| & \leq\left\|b_{j} \times u_{j} * \chi^{\delta_{j}}(\cdot, t)\right\|_{L^{1}(\Omega)}\|v\|_{L^{\infty}(\Omega)} \\
& +\mu_{j}\left\|\operatorname{curl} b_{j} * \chi^{\delta_{j}}(\cdot, t)\right\|_{L^{2}(\Omega)}\|v\|_{L^{2}(\Omega)}
\end{aligned}
$$

for all $v \in W_{0, \sigma}^{1,4}\left(\Omega^{\prime}, \mathbb{R}^{3}\right)$ so that, by Lemma 2.9 .

$$
\begin{aligned}
\left\|\partial_{t} \Psi_{j}^{\Sigma} * \chi^{\delta_{j}}\right\|_{L^{2}\left(\epsilon, T-\epsilon ;\left(W_{0, \sigma}^{1,4}(\Omega)\right)^{*}\right)} & \lesssim \Omega\left\|b_{j} \times u_{j} * \chi^{\delta_{j}}\right\|_{L^{2}\left(\epsilon, T-\epsilon ; L^{1}(\Omega)\right)} \\
& +\mu_{j}\left\|\operatorname{curl} b_{j} * \chi^{\delta_{j}}\right\|_{L^{2}\left(\epsilon, T-\epsilon ; L^{2}(\Omega)\right)} \\
& \leq\left\|b_{j} \times u_{j}\right\|_{L^{2}\left(0, T ; L^{1}(\Omega)\right)}+\mu_{j}\left\|\operatorname{curl} b_{j}\right\|_{L^{2}\left(0, T ; L^{2}(\Omega)\right)}
\end{aligned}
$$

which yields 4.7.

4.6. Completion of the proof. The proof of Theorem 1.5 will be finished in the following two lemmas by showing (4.4) and controlling the size of the integral term in 4.4. A third lemma then proves Corollary 1.6.

Lemma 4.5. For every $j \in \mathbb{N}$ and every $t \in[0, T)$ we have

$$
\mathscr{M}\left(b_{j} ; t\right)=\mathscr{M}\left(b_{j} ; 0\right)-2 \mu_{j} \int_{0}^{t} \int_{\Omega} b_{j}(x, \tau) \cdot \operatorname{curl} b_{j}(x, \tau) d x d \tau .
$$

Proof. We intend to show that

$$
\partial_{t} \mathscr{M}\left(b_{j} ; t\right)=-2 \mu_{j} \int_{\Omega} b_{j}(x, t) \cdot \operatorname{curl} b_{j}(x, t) d x
$$

in the sense of distributions; the claim then follows since the Cauchy-Schwarz inequality gives $\partial_{t} \mathscr{M}\left(b_{j} ; \cdot\right) \in L^{1}(0, T)$.

Let $\eta \in C_{c}^{\infty}(0, T)$ and note that $\int_{0}^{T} \eta(t) \mathscr{M}\left(b_{j} ; t\right) d t=\lim _{\delta \rightarrow 0} \int_{0}^{T} \eta(t) \mathscr{M}\left(b_{j, \delta} ; t\right) d t$ by Lemma 2.10 . Fix $\delta \in(0, T / 2)$ such that $\operatorname{supp}(\eta) \subset[2 \delta, T-2 \delta]$. Then, integrating by parts several times and using the facts that $\Psi_{j}^{\Sigma} \times\left. n\right|_{\Gamma}=0$ and $\partial_{t} \Psi_{j}^{H}=\partial_{t} b_{j, H}=0$ we get

$$
\begin{aligned}
\int_{0}^{T} \partial_{t} \eta(t) \mathscr{M}\left(b_{j, \delta} ; t\right) d t & =\int_{0}^{T} \partial_{t} \eta(t) \int_{\Omega}\left(\Psi_{j}^{\Sigma}+\psi_{j}^{H}\right)_{\delta}(x, t) \cdot\left(b_{j, \Sigma}+b_{j, H}\right)_{\delta}(x, t) d x d t \\
& =-2 \int_{0}^{T} \eta(t) \int_{\Omega} \partial_{t} \Psi_{j, \delta}^{\Sigma}(x, t) \cdot b_{j, \Sigma, \delta}(x, t) d x d t \\
& +2 \int_{0}^{T} \partial_{t} \eta_{\delta}(t) \int_{\Omega} \psi_{j}^{H}(x) \cdot b_{j, \Sigma}(x, t) d x d t=: I_{1}+I_{2} .
\end{aligned}
$$


For $I_{1}$ we note that $\partial_{t} \Psi_{j, \delta}^{\Sigma}+\left(b_{j} \times u_{j}\right)_{\delta}+\mu_{j} \operatorname{curl} b_{j, \delta} \in L^{\infty}(\delta, T-\delta ; \operatorname{ker}(\operatorname{curl}))=$ $L^{\infty}\left(\delta, T-\delta ;\left(L_{\Sigma}^{2}\left(\Omega, \mathbb{R}^{3}\right)\right)^{\perp}\right)$ so that

$$
I_{1}=2 \int_{0}^{T} \eta(t) \int_{\Omega}\left[\left(b_{j} \times u_{j}\right)_{\delta}(x, t)+\mu_{j} \operatorname{curl} b_{j, \delta}(x, t)\right] \cdot\left(b_{j, \delta}^{\Sigma}(x, t) d x d t .\right.
$$

For $I_{2}$ we note that since $\partial_{t}\left(\psi_{j}^{H} \cdot b_{j, H}\right)=0$, we get

$$
I_{2}=2 \int_{0}^{T} \partial_{t} \eta_{\delta}(t) \int_{\Omega} \psi_{j}^{H}(x) \cdot b_{j}(x, t) d x d t
$$

By setting $\theta(x, t):=2 \eta_{\delta}(t) \psi_{j}^{H}(x)$ in 2.5 we obtain

$$
\begin{aligned}
I_{2} & =2 \int_{0}^{T} \eta_{\delta}(t) \int_{\Omega} \operatorname{curl} \psi_{j}^{H}(x) \cdot\left[b_{j} \times u_{j}(x, t)+\mu_{j} \operatorname{curl} b_{j}(x, t)\right] d x d t \\
& =2 \int_{0}^{T} \eta(t) \int_{\Omega} b_{j}^{H}(x) \cdot\left[\left(b_{j} \times u_{j}\right)_{\delta}(x, t)+\mu_{j} \operatorname{curl} b_{j, \delta}(x, t)\right] d x d t .
\end{aligned}
$$

Collecting the identities, taking the limit $\delta \rightarrow 0$ (via Lemma 2.11) and using the pointwise identity $b_{j} \cdot b_{j} \times u_{j}=0$ we conclude that

$$
\int_{0}^{T} \partial_{t} \eta(t) \mathscr{M}\left(b_{j} ; t\right) d t=2 \mu_{j} \int_{0}^{T} \eta(t) \int_{\Omega} b_{j}(x, t) \cdot \operatorname{curl} b_{j}(x, t) d x,
$$

which yields 4.9 . 1.5 .

The following estimate, which goes back to 2, completes the proof of Theorem

Lemma 4.6. For every $j \in \mathbb{N}$,

$$
\mu_{j} \int_{0}^{T} \int_{\Omega}\left|b_{j}(x, t) \cdot \operatorname{curl} b_{j}(x, t)\right| d x d t \lesssim_{T} \sqrt{\mu_{j}}\left(\left\|u_{j, 0}\right\|_{L^{2}}^{2}+\left\|b_{j, 0}\right\|_{L^{2}}^{2}\right) .
$$

Proof. By Young's inequality and the energy inequality,

$$
\begin{aligned}
& \mu_{j} \int_{0}^{T} \int_{\Omega}\left|b_{j}(x, t) \cdot \operatorname{curl} b_{j}(x, t)\right| d x d t \\
\leq \quad & \frac{\sqrt{\mu_{j}}}{2} \int_{0}^{T} \int_{\Omega}\left(\left|b_{j}(x, t)\right|^{2}+\mu_{j}\left|\operatorname{curl} b_{j}(x, t)\right|^{2}\right) d x d t \\
\lesssim_{T} \quad & \sqrt{\mu_{j}}\left(\left\|u_{j, 0}\right\|_{L^{2}}^{2}+\left\|b_{j, 0}\right\|_{L^{2}}^{2}\right) .
\end{aligned}
$$

A simple lemma gives the rightmost equality in (1.13).

Lemma 4.7. $\lim _{j \rightarrow \infty} \int_{\Omega}\left(\Psi_{j, 0}^{\Sigma}(x)+\psi_{j, 0}^{H}(x)\right) \cdot b_{j, 0}(x) d x=\int_{\Omega}\left(\Psi_{j, 0}^{\Sigma}(x)+\psi_{j, 0}^{H}(x)\right) \cdot$ $b_{0}(x) d x$.

Proof. By assumption, $b_{j, 0} \rightarrow b_{0}$ in $L_{\sigma}^{2}\left(\Omega, \mathbb{R}^{3}\right)$, and therefore $\Psi_{j, 0}^{\Sigma}+\psi_{j, 0}^{H}=T_{\Sigma} b_{j, 0, \Sigma}+$ $T_{H} b_{j, 0, H} \rightarrow T_{\Sigma} b_{0, \Sigma}+T_{H} b_{0, H}=\Psi_{0}^{\Sigma}+\psi_{0}^{H}$ in $W^{1,2}\left(\Omega, \mathbb{R}^{3}\right)$. The Rellich-Kondrachov Theorem then yields $\left\|\left(\Psi_{j, 0}^{\Sigma}+\psi_{j, 0}^{H}\right)-\left(\Psi_{0}^{\Sigma}+\psi_{0}^{H}\right)\right\|_{L^{2}(\Omega)} \rightarrow 0$, which implies the claim. 


\section{A tWO-DIMENSIONAL ANALOGUE}

Magnetic helicity has a two-dimensional counterpart, the mean-square magnetic potential. It is defined as the $L^{2}$ energy of the canonical stream function of $b$, and it is conserved in time by smooth solutions of ideal 2D MHD. In $\$ 5.1$ we define the mean-square magnetic potential in multiply connected domains and formulate Theorem 5.4 which says that it is also conserved in the weak ideal limit. As a byproduct, we prove that if a weak solution of 2D ideal MHD lies in the energy space, then it conserves mean-square magnetic potential in time. The proof of Theorem 5.4 is presented in 55.2 . As main tools, apart from ones already used in 3D, we use C. Fefferman's $\mathcal{H}^{1}-\mathrm{BMO}$ duality theorem from [19] and the Hardy space theory of compensated compactness quantities of Coifman, Lions, Meyer and Semmes from [11.

5.1. Mean-square magnetic potential and statement of the theorem. In two dimensions, the viscous, resistive MHD equations are given by

$$
\begin{aligned}
& \partial_{t} u+(u \cdot \nabla) u-(b \cdot \nabla) b-\nu \Delta u+\nabla \Pi=0, \\
& \partial_{t} b-\nabla^{\perp}(b \times u)-\mu \nabla^{\perp}(\operatorname{curl} b)=0, \\
& \operatorname{div} u=\operatorname{div} b=0, \\
& u(\cdot, 0)=u_{0}, b(\cdot, 0)=b_{0},
\end{aligned}
$$

where $\nabla^{\perp}=\left(-\partial_{2}, \partial_{1}\right)$ and curl $=\nabla^{\perp}$. We now record our assumptions on the domain; we weaken the regularity condition that we placed on the boundary in three dimensions. Assumption 5.1 is strong enough to ensure the existence of a canonical stream function for every vector field in $L_{\sigma}^{2}\left(\Omega, \mathbb{R}^{2}\right)$ (see Theorem 5.2.

Assumption 5.1. The set $\Omega \subset \mathbb{R}^{2}$ is open and bounded. Furthermore, $\Omega$ is connected and its boundary $\Gamma$ is Lipschitz-continuous and has a finite number of connected components denoted by $\Gamma_{1}, \ldots, \Gamma_{K}$.

The boundary conditions corresponding to $1.5-1.6$ are

$$
\begin{aligned}
& \left.u\right|_{\Gamma}=0, \\
& \left.b \cdot n\right|_{\Gamma}=0 \quad \text { and }\left.\quad \operatorname{curl} b\right|_{\Gamma}=0 .
\end{aligned}
$$

Equations 5.1 -5.6 are understood in analogy to the 3D case, but 2.4 needs to be replaced by the formula

$$
\left\langle\partial_{t} b, \theta\right\rangle+\int_{\Omega} b \times u \operatorname{curl} \theta+\mu \int_{\Omega} \operatorname{curl} b \operatorname{curl} \theta=0 .
$$

We enumerate $\Gamma_{1}, \ldots, \Gamma_{K}$ in such a way that $\Gamma_{1}$ is the boundary of the unbounded component of $\mathbb{R}^{2} \backslash \bar{\Omega}$. Following [27, p. 40] we denote

$$
\Phi:=\left\{\psi \in W^{1,2}(\Omega):\left.\psi\right|_{\Gamma_{1}}=0,\left.\psi\right|_{\Gamma_{i}} \text { is constant for } 2 \leq i \leq K\right\} ;
$$

note that if $\Gamma$ is connected, then $\Phi=W_{0}^{1,2}(\Omega)$. The following theorem gives a canonical choice of stream functions (see [27, Corollary I.3.1]).

Theorem 5.2. The mapping $-\nabla^{\perp}: \Phi \rightarrow L_{\sigma}^{2}\left(\Omega, \mathbb{R}^{2}\right)$ is an isomorphism.

When $v \in L_{\sigma}^{2}\left(\Omega, \mathbb{R}^{2}\right)$, we call $\left(-\nabla^{\perp}\right)^{-1} v \in \Phi$ the stream function of $v$. LerayHopf solutions are defined in direct analogy to Definition 2.13 . 
Definition 5.3. Suppose $(u, b)$ is a Leray-Hopf solution of (5.1) 5.6 and $\psi \in$ $C_{w}([0, T) ; \Phi)$ is the stream function of $b$. For every $t \in[0, T), \int_{\Omega}|\psi(x, t)|^{2} d x$ is called the mean-square magnetic potential of $b$ at time $t$.

We formulate an analogue of Theorem 1.5 for the mean-square magnetic potential, denoting the stream functions of the initial datas $b_{j, 0}$ and $b_{0}$ by $\psi_{j, 0}$ and $\psi_{0}$. The weak ideal limit and weak non-resistive limit are defined in direct analogy to Definition 1.1.

Theorem 5.4. Suppose $\Omega \subset \mathbb{R}^{2}$ satisfies Assumption 5.1, and assume that $u, b \in$ $L^{\infty}\left(0, T ; L_{\sigma}^{2}\left(\Omega, \mathbb{R}^{3}\right)\right)$ are a weak ideal limit or weak non-resistive limit of Leray-Hopf solutions $\left(u_{j}, b_{j}\right), j \in \mathbb{N}$. Then $b \in C_{w}\left([0, T) ; L_{\sigma}^{2}\left(\Omega, \mathbb{R}^{2}\right)\right), \partial_{t} b-\nabla^{\perp}(b \times u)=0$ with $b(\cdot, 0)=b_{0}$ and

$$
\int_{\Omega}|\psi(x, t)|^{2} d x=\int_{\Omega}\left|\psi_{0}(x)\right|^{2} d x=\lim _{j \rightarrow \infty} \int_{\Omega}\left|\psi_{j, 0}(x)\right|^{2} d x
$$

for all $t \in[0, T)$.

Note that Theorem 5.4 is stronger than Theorem 1.5 in the sense that the induction equation $\partial_{t} b-\nabla^{\perp}(b \times u)=0$ holds in the weak ideal limit. Theorem 5.4 is proved in the following subsection.

5.2. Proof of Theorem 5.4. Our first task is to prove that the induction equation $\partial_{t} b-\nabla^{\perp}(b \times u)=0$ holds and $b(\cdot, 0)=b_{0}$. We begin the proof by showing a $2 \mathrm{D}$ analogue of Lemma 4.4

Lemma 5.5. $\psi_{j} \rightarrow \psi$ in $L^{2}\left(0, T ; L^{2}(\Omega)\right)$.

Proof. Since $-\nabla^{\perp} \psi_{j}=b_{j} \rightarrow b=-\nabla^{\perp} \psi$ in $L^{2}\left(0, T ; L_{\sigma}^{2}\left(\Omega, \mathbb{R}^{2}\right)\right)$, we have $\psi^{j} \rightarrow \psi$ in $L^{2}(0, T ; \Phi)$ by Theorem 5.2 . Hence, it suffices, by the Aubin-Lions Lemma, to show that

$$
\sup _{j \in \mathbb{N}}\left\|\partial_{t} \psi_{j}\right\|_{L^{1}\left(0, T ; W^{-1,2}(\Omega)\right)}<\infty .
$$

We write $\partial_{t} \psi_{j}=-b_{j} \times u_{j}-\mu_{j} \operatorname{curl} b_{j}$ and estimate the terms separately. First, we set

$$
B_{j}(x, t):=\left\{\begin{array}{ll}
b_{j}(x, t), & x \in \Omega, \\
0, & x \notin \Omega,
\end{array} \quad U_{j}(x, t):= \begin{cases}u_{j}(x, t), & x \in \Omega, \\
0, & x \notin \Omega\end{cases}\right.
$$

and note that $\operatorname{div} B_{j}=\operatorname{div} U_{j}=0$ in $\mathbb{R}^{2} \times(0, T)$. Fix $t \in(0, T)$ and $\varphi \in C_{c}^{\infty}(\Omega)$, and denote $\Phi(x)=\varphi(x)$ for $x \in \Omega$ and $\Phi(x)=0$ for $x \notin \Omega$. Fefferman's $\mathcal{H}^{1}$-BMO duality theorem and the div-curl estimate of Coifman, Lions, Meyer and Semmes give

$$
\begin{aligned}
\int_{\Omega} b_{j}(x, t) \times u_{j}(x, t) \varphi(x) d x & =\int_{\mathbb{R}^{2}} B_{j}(x, t) \times U_{j}(x, t) \Phi(x) d x \\
& \lesssim\left\|B_{j}(\cdot, t) \times U_{j}(\cdot, t)\right\|_{\mathcal{H}^{1}}\|\Phi\|_{\mathrm{BMO}} \\
& \lesssim\left\|B_{j}(\cdot, t)\right\|_{L^{2}}\left\|U_{j}(\cdot, t)\right\|_{L^{2}}\|\nabla \Phi\|_{L^{2}},
\end{aligned}
$$

yielding, by the Cauchy-Schwartz inequality,

$$
\sup _{j \in \mathbb{N}}\left\|b_{j} \times u_{j}\right\|_{L^{1}\left(0, T ; W^{-1,2}(\Omega)\right)} \lesssim \sup _{j \in \mathbb{N}}\left\|b_{j}\right\|_{L^{2}\left(0, T ; L^{2}(\Omega)\right)}\left\|u_{j}\right\|_{L^{2}\left(0, T ; L^{2}(\Omega)\right)}<\infty .
$$

Furthermore, trivially, $\sup _{j \in \mathbb{N}}\left\|\mu_{j} \operatorname{curl} b_{j}\right\|_{L^{1}\left(0, T ; W^{-1,2}(\Omega)\right)}<\infty$, and so $(5.9)$ holds. 
We next show that the limit mappings $u$ and $b$ satisfy the ideal momentum equation. Given $j \in \mathbb{N}$, the mappings $u_{j}$ and $b_{j}$ satisfy (5.1)-5.6 and using standard arguments (see e.g. [24, Lemma 2.4]), (5.7) and the initial value condition $b_{j}(\cdot, 0)=b_{j, 0}$ yield

$$
\int_{0}^{T} \int_{\Omega}\left(b_{j} \cdot \partial_{t} \phi-b_{j} \times u_{j} \operatorname{curl} \phi-\mu \operatorname{curl} b_{j} \operatorname{curl} \phi\right) d x d t+\int_{\Omega} b_{j, 0} \cdot \phi(\cdot, 0)
$$

for every $\phi \in C_{c}^{\infty}\left(\Omega \times[0, T), \mathbb{R}^{2}\right)$ with $\operatorname{div} \phi=0$.

Lemma 5.6. $\partial_{t} b-\nabla^{\perp}(b \times u)=0$ with initial value $b(\cdot, 0)=b_{0}$.

Proof. Fix $\phi \in C_{c}^{\infty}\left(\Omega \times[0, T), \mathbb{R}^{2}\right)$ with $\operatorname{div} \phi=0$. By using the formula $b_{j} \times u_{j}=$ $\nabla \psi_{j} \cdot u_{j}$, Theorem 3.1 and Lemma 5.5.

$$
\begin{aligned}
& \int_{0}^{T} \int_{\Omega} b_{j}(x, t) \times u_{j}(x, t) \operatorname{curl} \phi(x, t) d x d t \\
= & \int_{0}^{T} \int_{\Omega} \nabla\left[\operatorname{curl} \phi(x, t) \psi_{j}(x, t)\right] \cdot u_{j}(x, t) d x d t \\
- & \int_{0}^{T} \int_{\Omega} \psi_{j}(x, t) \nabla \operatorname{curl} \phi(x, t) \cdot u_{j}(x, t) d x d t \\
\rightarrow & -\int_{0}^{T} \int_{\Omega} \psi(x, t) \nabla \operatorname{curl} \phi(x, t) \cdot u(x, t) d x d t \\
= & \int_{0}^{T} \int_{\Omega} b(x, t) \times u(x, t) \operatorname{curl} \phi(x, t) d x d t .
\end{aligned}
$$

The claim now follows immediately by inspection of 5.11 , since the energy inequality yields $\mu_{j} \int_{0}^{T} \int_{\Omega} \operatorname{curl} b_{j}(x, t) \operatorname{curl} \phi(x, t) d x d t \rightarrow 0$.

As in 3D, by adapting [24, Lemmas 2.1 and 2.2] we may assume that $b \in$ $C_{w}\left([0, T) ; L_{\sigma}^{2}\left(\Omega, \mathbb{R}^{2}\right)\right)$. Thus the stream function $\psi$ belongs to $C_{w}([0, T) ; \Phi) \subset$ $C\left([0, T) ; L^{2}(\Omega)\right)$.

Theorem 5.4 will be proved once we show (5.8). The right equality in (5.8) follows from the assumption $b_{j, 0} \rightarrow b_{0}$ and the Rellich-Kondrachov Theorem. We next prove the left equality - in fact, we also prove that every weak solution of ideal MHD in the energy space conserves magnetic helicity in time.

Lemma 5.7. Suppose $u \in L^{\infty}\left(0, T ; L_{\sigma}^{2}\left(\Omega, \mathbb{R}^{2}\right)\right)$ and $b \in C_{w}\left([0, T) ; L_{\sigma}^{2}\left(\Omega, \mathbb{R}^{2}\right)\right)$ satisfy $\partial_{t} b-\nabla^{\perp}(b \times u)=0$ with initial value $b(\cdot, 0)=b_{0} \in L_{\sigma}^{2}\left(\Omega, \mathbb{R}^{2}\right)$. Then $b$ conserves mean square magnetic potential in time.

Proof. Since $\psi \in C\left([0, T) ; L^{2}(\Omega)\right)$, it suffices to show that for every $\eta \in C_{c}^{\infty}(0, T)$ we have $\int_{0}^{T} \partial_{t} \eta(t) \int_{\Omega}|\psi(x, t)|^{2} d x d t=0$. Fix such an $\eta$ and choose $\epsilon>0$ such that $\operatorname{supp}(\eta) \subset[\epsilon, T-\epsilon]$. Whenever $0<\delta<\epsilon$, we mollify in time and write $\psi_{\delta}:=\psi * \chi_{\delta}$. By Lemma 2.10, $\int_{0}^{T} \partial_{t} \eta(t) \int_{\Omega}|\psi(x, t)|^{2} d x d t=\lim _{\delta \rightarrow 0} \int_{0}^{T} \partial_{t} \eta(t) \int_{\Omega}\left|\psi_{\delta}(x, t)\right|^{2} d x d t$.

Let now $0<\delta<\epsilon$. The induction equation $\partial_{t} b-\nabla^{\perp}(b \times u)=0$ and the assumptions about the boundary values of $u, b$ and $\psi$ imply that $\partial_{t} \psi+b \times u=0$, and thus $(b \times u)_{\delta}=-\psi * \partial_{t} \chi_{\delta} \in L^{\infty}\left(\epsilon, T-\epsilon ; W^{1,2}(\Omega)\right)$, giving

$$
\int_{0}^{T} \partial_{t} \eta(t) \int_{\Omega}\left|\psi_{\delta}(x, t)\right|^{2} d x d t=2 \int_{0}^{T} \eta(t) \int_{\Omega} \psi_{\delta}(x, t)(b \times u)_{\delta}(x, t) d x d t .
$$


As in (5.10), we denote the zero extensions of $b$ and $u$ outside $\Omega$ by $B$ and $U$. Likewise, for every $t \in[0, T)$, we denote by $\Psi(\cdot, t) \in W^{1,2}\left(\mathbb{R}^{2}\right)$ the unique compactly supported solution of $-\nabla^{\perp} \Psi(\cdot, t)=B(\cdot, t)$. Thus $\Psi \in L^{\infty}\left(0, T ; W^{1,2}\left(\mathbb{R}^{2}\right)\right) \subset$ $L^{2}\left(0, T ; \operatorname{VMO}\left(\mathbb{R}^{2}\right)\right)$ and $B \times U \in L^{\infty}\left(0, T ; \mathcal{H}^{1}\left(\mathbb{R}^{2}\right)\right) \subset\left(L^{2}\left(0, T ; \operatorname{VMO}\left(\mathbb{R}^{2}\right)\right)\right)^{*}$. This allows us to write, using Lemma 2.10 .

$$
\begin{aligned}
\int_{0}^{T} \eta(t) \int_{\Omega} \psi_{\delta}(x, t)(b \times u)_{\delta}(x, t) d x d t & =\int_{0}^{T} \eta(t) \int_{\mathbb{R}^{2}} \Psi_{\delta}(x, t)(B \times U)_{\delta}(x, t) d x d t \\
& \rightarrow \int_{0}^{T} \eta(t)\langle\Psi(\cdot, t), B \times U(\cdot, t)\rangle_{\mathrm{VMO}-\mathcal{H}^{1}} d t .
\end{aligned}
$$

We finally mollify $\Psi$ and $B$ in space and use the Dominated Convergence Theorem in time to conclude that

$$
\begin{aligned}
& \int_{0}^{T} \eta(t)\langle\Psi(\cdot, t), B \times U(\cdot, t)\rangle_{\mathrm{VMO}-\mathcal{H}^{1}} d t \\
= & \lim _{\epsilon \rightarrow 0} \int_{0}^{T} \eta(t) \int_{\mathbb{R}^{2}} \Psi_{\epsilon}(x, t) B_{\epsilon}(x, t) \times U(x, t) d x d t \\
= & \frac{1}{2} \lim _{\epsilon \rightarrow 0} \int_{0}^{T} \eta(t) \int_{\mathbb{R}^{2}} \nabla\left|\Psi_{\epsilon}(x, t)\right|^{2} \cdot U(x, t) d x d t=0 .
\end{aligned}
$$

\section{Appendix A. The existence of Leray-Hopf solutions in MUltiply CONNECTED DOMAINS}

We give a proof of the existence of Leray-Hopf solutions of (1.1)-(1.6), referring to the literature on some of the steps that are familiar from Navier-Stokes equations. A proof for simply connected domains is sketched in 44] and presented in more detail in [26]. As we cover multiply connected domains, more technicalities are needed although we follow the general scheme of the proof given in [26. We reformulate Theorem 2.14 for the convenience of the reader.

Theorem A.1. Suppose $\Omega$ satisfies Assumption 2.1 and let $u_{0}, b_{0} \in L_{\sigma}^{2}\left(\Omega, \mathbb{R}^{3}\right)$. Then there exists a Leray-Hopf solution $(u, b)$ of $(1.1)-(1.6)$.

The basic strategy of the proof, via finite-dimensional Galerkin approximations, is classical, but we discuss the main ideas. The solution is built via orthonormal bases $\left\{v_{j}\right\}_{j \in \mathbb{N}}$ and $\left\{w_{j}\right\}_{j \in \mathbb{N}}$ of $L_{\sigma}^{2}\left(\Omega, \mathbb{R}^{3}\right)$ satisfying the sought boundary conditions, that is, $v_{j} \in W_{0, \sigma}^{1,2}\left(\Omega, \mathbb{R}^{3}\right)$ and $w_{j} \in W_{\sigma}^{1,2}\left(\Omega, \mathbb{R}^{3}\right)$ with $\left.\left(\operatorname{curl} w_{j} \times n\right)\right|_{\Gamma}=0$.

Definition A.2. Suppose $u_{0}, b_{0} \in L_{\sigma}^{2}\left(\Omega, \mathbb{R}^{3}\right)$ and let $n \in \mathbb{N}$. Mappings of the forms

$$
u_{n}(x, t)=\sum_{j=1}^{n} c_{n j}(t) v_{j}(x), \quad b_{n}(x, t)=\sum_{j=1}^{n} d_{n j}(t) w_{j}(x),
$$

where $c_{n j}, d_{n j} \in C^{1}([0, T))$, satisfy the $n$th order Galerkin approximation of 1.1 (1.6) if

$$
\begin{aligned}
& \frac{d}{d t}\left(u_{n}, v_{j}\right)_{L^{2}}+\nu\left(\nabla u_{n}, \nabla v_{j}\right)_{L^{2}}+\left\langle\left(u_{n} \cdot \nabla\right) u_{n}-\left(b_{n} \cdot \nabla\right) b_{n}, v_{j}\right\rangle_{\left(W_{0, \sigma}^{1,2}\right)^{*}-W_{0, \sigma}^{1,2}}=0, \\
& \frac{d}{d t}\left(b_{n}, w_{j}\right)_{L^{2}}+\mu\left(\operatorname{curl} b_{n}, \operatorname{curl} w_{j}\right)_{L^{2}}+\left\langle\operatorname{curl}\left(b_{n} \times u_{n}\right), w_{j}\right\rangle_{\left(W_{\sigma}^{1,2}\right)^{*}-W_{\sigma}^{1,2}}=0, \\
& u_{n}(\cdot, 0)=P_{n} u_{0}, \quad b_{n}(\cdot, 0)=Q_{n} b_{0}
\end{aligned}
$$


for all $j=1, \ldots, n$.

For every $n \in \mathbb{N}$, standard theory of ordinary differential equations gives a unique solution of the Galerkin approximation satisfying the energy equality

$$
\begin{aligned}
& \frac{1}{2} \int_{\Omega}\left(\left|u_{n}(x, t)\right|^{2}+\left|b_{n}(x, t)\right|^{2}\right) d x \\
+ & \int_{0}^{t} \int_{\Omega}\left(\nu\left|\nabla u_{n}(x, \tau)\right|^{2}+\mu\left|\operatorname{curl} b_{n}(x, \tau)\right|^{2}\right) d x d \tau \\
= & \frac{1}{2} \int_{\Omega}\left(\left|P_{n} u_{0}(x)\right|^{2}+\left|Q_{n} b_{0}(x)\right|^{2}\right) d x
\end{aligned}
$$

for all $t \in(0, T)$ (see Lemma A.8). With some work, the energy equality allows us to subtract a subsequence with $u_{n} \rightarrow u$ in $L^{2}\left(0, T ; W_{0, \sigma}^{1,2}\left(\Omega, \mathbb{R}^{3}\right)\right)$ and $b_{n} \rightarrow b$ in $L^{2}\left(0, T ; W_{\sigma}^{1,2}\left(\Omega, \mathbb{R}^{3}\right)\right)$. Our goal is to show that $(u, b)$ is a Leray-Hopf solution with initial data $\left(u_{0}, b_{0}\right)$.

For every $n \in \mathbb{N}$ we denote by $P_{n}$ and $Q_{n}$ the projections of $L_{\sigma}^{2}\left(\Omega, \mathbb{R}^{3}\right)$ onto $\operatorname{span}\left\{v_{1}, \ldots, v_{n}\right\}$ and $\operatorname{span}\left\{w_{1}, \ldots, w_{n}\right\}$. Note that $P_{n}: W_{0, \sigma}^{1,2}\left(\Omega, \mathbb{R}^{3}\right) \rightarrow W_{0, \sigma}^{1,2}\left(\Omega, \mathbb{R}^{3}\right)$ and $Q_{n}: W_{\sigma}^{1,2}\left(\Omega, \mathbb{R}^{3}\right) \rightarrow W_{\sigma}^{1,2}\left(\Omega, \mathbb{R}^{3}\right)$ are also bounded operators, and we denote their (Banach space) adjoints by $P_{n}^{*}:\left(W_{0, \sigma}^{1,2}\left(\Omega, \mathbb{R}^{3}\right)\right)^{*} \rightarrow\left(W_{0, \sigma}^{1,2}\left(\Omega, \mathbb{R}^{3}\right)\right)^{*}$ and $Q_{n}^{*}:\left(W_{\sigma}^{1,2}\left(\Omega, \mathbb{R}^{3}\right)\right)^{*} \rightarrow\left(W_{\sigma}^{1,2}\left(\Omega, \mathbb{R}^{3}\right)\right)^{*}$. We also define the Stokes operator and a corresponding operator for magnetic fields,

$$
\Lambda_{1}: W_{0, \sigma}^{1,2}\left(\Omega, \mathbb{R}^{3}\right) \rightarrow\left(W_{0, \sigma}^{1,2}\left(\Omega, \mathbb{R}^{3}\right)\right)^{*} \quad \text { and } \quad \Lambda_{2}: W_{\sigma}^{1,2}\left(\Omega, \mathbb{R}^{3}\right) \rightarrow\left(W_{\sigma}^{1,2}\left(\Omega, \mathbb{R}^{3}\right)\right)^{*},
$$

by

$$
\begin{aligned}
\left\langle\Lambda_{1} u, v\right\rangle_{\left(W_{0, \sigma}^{1,2}\right)^{*}-W_{0, \sigma}^{1,2}}:=\int_{\Omega} \nabla u: \nabla v, \\
\left\langle\Lambda_{2} b, w\right\rangle_{\left(W_{\sigma}^{1,2}\right)^{*}-W_{\sigma}^{1,2}}:=\int_{\Omega} \operatorname{curl} b \cdot \operatorname{curl} w .
\end{aligned}
$$

We write the Galerkin approximation in the condensed form

$$
\begin{aligned}
& \partial_{t} u_{n}-\nu P_{n}^{*} \Lambda_{1} u_{n}+P_{n}^{*}\left[\left(u_{n} \cdot \nabla\right) u_{n}-\left(b_{n} \cdot \nabla\right) b_{n}\right]=0, \\
& \partial_{t} b_{n}-\mu Q_{n}^{*} \Lambda_{2} b_{n}+Q_{n}^{*}\left[\operatorname{curl}\left(b_{n} \times u_{n}\right)\right]=0, \\
& u_{n}(\cdot, 0)=P_{n} u_{0}, \quad b_{n}(\cdot, 0)=Q_{n} b_{0} .
\end{aligned}
$$

In order for the weak limit $(u, b)$ to satisfy the MHD equations 1.1$)-(1.6)$ we need to gain enough compactness in the nonlinear terms $P_{n}^{*}\left[\left(u_{n} \cdot \nabla\right) \cdot u_{n}-b_{n} \times\left(\operatorname{curl} b_{n}\right)\right]$ and $Q_{n}^{*}\left[\operatorname{curl}\left(b_{n} \times u_{n}\right)\right]$. This is eventually achieved by using the Aubin Lions Lemma to get $u_{n} \rightarrow u$ and $b_{n} \rightarrow b$ in $L^{2}\left(0, T ; L_{\sigma}^{2}\left(\Omega, \mathbb{R}^{3}\right)\right)$. In order to satisfy the assumptions of the Aubin-Lions Lemma we wish to choose suitable bases $\left\{v_{j}\right\}_{j \in \mathbb{N}}$ and $\left\{w_{j}\right\}_{j \in \mathbb{N}}$ (see A.1) that ensure the uniform norm control

$$
\sup _{n \in \mathbb{N}}\left(\left\|P_{n}^{*}\right\|_{\left(W_{0, \sigma}^{1,2}\left(\Omega, \mathbb{R}^{3}\right)\right)^{*} \rightarrow\left(W_{0, \sigma}^{1,2}\left(\Omega, \mathbb{R}^{3}\right)\right)^{*}}+\left\|Q_{n}^{*}\right\|_{\left(W_{\sigma}^{1,2}\left(\Omega, \mathbb{R}^{3}\right)\right)^{*} \rightarrow\left(W_{\sigma}^{1,2}\left(\Omega, \mathbb{R}^{3}\right)\right)^{*}}\right)<\infty .
$$

As is customary, we select $v_{j}$ to be eigenfunctions of $\Lambda_{1}$, while a basis of $L_{\Sigma}^{2}\left(\Omega, \mathbb{R}^{3}\right)$ consists of eigenfunctions of $\Lambda_{2}$. Since we deal with multiply connected domains, $\left\{w_{j}\right\}_{j \in \mathbb{N}}$ also needs to include a basis of $L_{H}^{2}\left(\Omega, \mathbb{R}^{3}\right)$, and some care is needed in the ensuing arguments. The proof is completed in $\mathrm{A} .3$. 
A.1. The choice of bases. This subsection is devoted to the choice of the orthonormal bases of $L_{\sigma}^{2}\left(\Omega, \mathbb{R}^{3}\right)$. The first basis $\left\{v_{j}\right\}_{j \in \mathbb{N}}$ consists of eigenfunctions of the Stokes operator and its existence is classical; we refer to [47, p. 39]. We endow $W_{0, \sigma}^{1,2}\left(\Omega, \mathbb{R}^{3}\right)$ with the Hilbert norm $\|\cdot\|_{W_{0, \sigma}^{1,2}}:=\|\nabla \cdot\|_{L^{2}}$.

Lemma A.3. $L_{\sigma}^{2}\left(\Omega, \mathbb{R}^{3}\right)$ has an orthonormal basis $\left\{v_{j}\right\}_{j \in \mathbb{N}}$ with the following properties: for every $j \in \mathbb{N}$ there exists $\lambda_{j}>0$ such that $v_{j} \in W_{0, \sigma}^{1,2}\left(\Omega, \mathbb{R}^{3}\right)$ satisfies

$$
\left(v_{j}, \phi\right)_{W_{0, \sigma}^{1,2}}=\lambda_{j}\left(v_{j}, \phi\right)_{L^{2}}
$$

for all $\phi \in W_{0, \sigma}^{1,2}\left(\Omega, \mathbb{R}^{3}\right)$. In particular, $\left\{v_{j} / \sqrt{\lambda_{j}}\right\}_{j \in \mathbb{N}}$ is an orthonormal system in $W_{0, \sigma}^{1,2}\left(\Omega, \mathbb{R}^{3}\right)$.

The analysis of the second basis is simplified by using the following lemma which is essentially a special case of [1, Corollary 3.16].

Lemma A.4. On $W_{\sigma}^{1,2}\left(\Omega, \mathbb{R}^{3}\right)$, the norm $\|\cdot\|_{W_{\sigma}^{1,2}}$ induced by the inner product

$$
(v, w)_{W_{\sigma}^{1,2}}:=\int_{\Omega} \operatorname{curl} v(x) \cdot \operatorname{curl} w(x) d x+\sum_{i=1}^{N} \gamma_{i}\langle v \cdot n, 1\rangle_{\Sigma_{i}}\langle w \cdot n, 1\rangle_{\Sigma_{i}}
$$

(where $\gamma_{i}>0$ is chosen such that $\left\|h_{i}\right\|_{W_{\sigma}^{1,2}}=1$ for all $i \in\{1, \ldots, N\}$ ) is equivalent to the norm inherited from $W^{1,2}\left(\Omega, \mathbb{R}^{3}\right)$.

Lemma A.4 has the following consequence ( [15, Lemme II.6]).

Lemma A.5. The vector spaces $W_{\Sigma}^{1,2}\left(\Omega, \mathbb{R}^{3}\right):=W_{\sigma}^{1,2}\left(\Omega, \mathbb{R}^{3}\right) \cap L_{\Sigma}^{2}\left(\Omega, \mathbb{R}^{3}\right)$ and $W_{H}^{1,2}\left(\Omega, \mathbb{R}^{3}\right):=W_{\sigma}^{1,2}\left(\Omega, \mathbb{R}^{3}\right) \cap L_{H}^{2}\left(\Omega, \mathbb{R}^{3}\right)$ satisfy

$$
W_{\sigma}^{1,2}\left(\Omega, \mathbb{R}^{3}\right)=W_{\Sigma}^{1,2}\left(\Omega, \mathbb{R}^{3}\right) \oplus W_{H}^{1,2}\left(\Omega, \mathbb{R}^{3}\right) .
$$

Furthermore, $W_{\Sigma}^{1,2}\left(\Omega, \mathbb{R}^{3}\right)$ is dense in $L_{\Sigma}^{2}\left(\Omega, \mathbb{R}^{3}\right)$.

Proof. In the proof of A.7) the only non-trivial condition to check is that when $w \in$ $W_{\sigma}^{1,2}\left(\Omega, \mathbb{R}^{3}\right)$, we have $w_{\Sigma}:=P_{\Sigma} w \in W_{\Sigma}^{1,2}\left(\Omega, \mathbb{R}^{3}\right)$ and $w_{H}:=P_{H} w \in W_{H}^{1,2}\left(\Omega, \mathbb{R}^{3}\right)$. Note that Theorem 2.8 gives $w_{H} \in W_{H}^{1,2}\left(\Omega, \mathbb{R}^{3}\right)$, which immediately implies $w_{\Sigma} \in$ $W_{\Sigma}^{1,2}\left(\Omega, \mathbb{R}^{3}\right)$. Furthermore, the projection $P_{\Sigma}: L_{\sigma}^{2}\left(\Omega, \mathbb{R}^{3}\right) \rightarrow L_{\Sigma}^{2}\left(\Omega, \mathbb{R}^{3}\right)$ is also a bounded operator from $W_{\sigma}^{1,2}\left(\Omega, \mathbb{R}^{3}\right)$ into $W_{\Sigma}^{1,2}\left(\Omega, \mathbb{R}^{3}\right)$.

Let now $f \in L_{\Sigma}^{2}\left(\Omega, \mathbb{R}^{3}\right)$ and choose mappings $\psi_{j} \in C_{c, \sigma}^{\infty}\left(\Omega, \mathbb{R}^{3}\right) \subset W_{\sigma}^{1,2}\left(\Omega, \mathbb{R}^{3}\right)$ such that $\left\|\psi_{j}-f\right\|_{L^{2}} \rightarrow 0$. Then $P_{\Sigma} \psi_{j} \in W_{\Sigma}^{1,2}\left(\Omega, \mathbb{R}^{3}\right)$ for all $j \in \mathbb{N}$ and $P_{\Sigma} \psi_{j} \rightarrow$ $P_{\Sigma} f=f$ in $L^{2}\left(\Omega, \mathbb{R}^{3}\right)$.

We use Lemma A.5 to find the basis of $L_{\sigma}^{2}\left(\Omega, \mathbb{R}^{3}\right)$ that is used to construct the magnetic field in Theorem A.1. In the case of simply connected domains this is done by analysing the magnetostatic problem instead of the stationary Stokes problem (see [26, pp. 67-69]). In multiply connected domains the situation is a bit more complicated because $W_{H}^{1,2}\left(\Omega, \mathbb{R}^{3}\right)$ is non-trivial.

Lemma A.6. $L_{\sigma}^{2}\left(\Omega, \mathbb{R}^{3}\right)$ has an orthonormal basis $\left\{w_{j}\right\}_{j \in \mathbb{N}}$ with the following properties: $\left\{w_{1}, \ldots, w_{N}\right\}=\left\{h_{1}, \ldots, h_{N}\right\}$ and for every $j \in \mathbb{N}$ there exists $\tilde{\lambda}_{j}>0$ such that $w_{j} \in W_{\sigma}^{1,2}\left(\Omega, \mathbb{R}^{3}\right)$ satisfies

$$
\left(w_{j}, \psi\right)_{W_{\sigma}^{1,2}}=\tilde{\lambda}_{j}\left(w_{j}, \psi\right)_{L^{2}}
$$


for all $\psi \in W_{\sigma}^{1,2}\left(\Omega, \mathbb{R}^{3}\right)$. In particular, $\left\{w_{j} /{\sqrt{\tilde{\lambda}_{j}}}_{j \in \mathbb{N}}\right.$ is an orthonormal system in $W_{\sigma}^{1,2}\left(\Omega, \mathbb{R}^{3}\right)$.

Proof. Given $j \in\{1, \ldots, N\}$ we first check that A.8 holds for $w_{j}=h_{j}$ with $\tilde{\lambda}_{j}=1$. Let $\psi \in W_{\sigma}^{1,2}\left(\Omega, \mathbb{R}^{3}\right)$. Writing $\psi=\sum_{i=1}^{N}\left(\psi, h_{i}\right)_{L^{2}} h_{i}+\psi_{\Sigma}$ and using Lemma A.5. (A.8) follows immediately.

Next we set out to find $w_{j} \in W_{\Sigma}^{1,2}\left(\Omega, \mathbb{R}^{3}\right)$ for every $j>N$. Given $f \in L_{\Sigma}^{2}\left(\Omega, \mathbb{R}^{3}\right)$ we define a quadratic functional $K: W_{\Sigma}^{1,2}\left(\Omega, \mathbb{R}^{3}\right) \rightarrow \mathbb{R}$ by

$$
K(C):=\frac{1}{2} \int_{\Omega}|\operatorname{curl} C(x)|^{2} d x-\int_{\Omega} f(x) \cdot C(x) d x .
$$

The quadratic part of $K$ is coercive, and therefore $K$ has a unique minimizer $w$ in $W_{\Sigma}^{1,2}\left(\Omega, \mathbb{R}^{3}\right)$. Thus

$$
\int_{\Omega} \operatorname{curl} w(x) \cdot \operatorname{curl} \psi(x) d x=\int_{\Omega} f(x) \cdot \psi(x) d x
$$

for all $\psi \in W_{\Sigma}^{1,2}\left(\Omega, \mathbb{R}^{3}\right)$.

We define a bounded linear operator $\mathcal{A}_{2}: L_{\Sigma}^{2}\left(\Omega, \mathbb{R}^{3}\right) \rightarrow L_{\Sigma}^{2}\left(\Omega, \mathbb{R}^{3}\right)$ by $\mathcal{A}_{2} f:=w$. Our aim is to choose $w_{j}, j>N$, as eigenfunctions of $\mathcal{A}_{2}$. Since

$$
\|w\|_{W^{1,2}(\Omega)}^{2} \lesssim \Omega\|\operatorname{curl} w\|_{L^{2}(\Omega)}^{2}=\int_{\Omega} f \cdot w \leq\|f\|_{L^{2}(\Omega)}\|w\|_{L^{2}(\Omega)}
$$

for all $f \in L_{\Sigma}^{2}\left(\Omega, \mathbb{R}^{3}\right)$, the Rellich-Kondrachov Theorem implies that $\mathcal{A}_{2}$ is compact. In addition, $\int_{\Omega} \mathcal{A}_{2} f(x) \cdot g(x) d x=\int_{\Omega} \operatorname{curl} \mathcal{A}_{2} f(x) \cdot \operatorname{curl} \mathcal{A}_{2} g(x) d x=\int_{\Omega} f(x)$. $\mathcal{A}_{2} g(x) d x$ for all $f, g \in L_{\Sigma}^{2}\left(\Omega, \mathbb{R}^{3}\right)$ so that $\mathcal{A}_{2}$ is self-adjoint. Furthermore, $\mathcal{A}_{2}$ is a positive operator. Indeed, given $f \in L_{\Sigma}^{2}\left(\Omega, \mathbb{R}^{3}\right) \backslash\{0\}$ we write $\int_{\Omega} \mathcal{A}_{2} f(x) \cdot f(x) d x=$ $\left\|\operatorname{curl} \mathcal{A}_{2} f\right\|_{L^{2}(\Omega)}^{2}$. Now A.9 and the assumption $f \neq 0$ imply that $\operatorname{curl} \mathcal{A}_{2} f \neq 0$ : since $W_{\Sigma}^{1,2}\left(\Omega, \mathbb{R}^{3}\right)$ is dense in $L_{\Sigma}^{2}\left(\Omega, \mathbb{R}^{3}\right)$ by Lemma A.5. we may choose $\psi \in$ $W_{\Sigma}^{1,2}\left(\Omega, \mathbb{R}^{3}\right)$ such that $\int_{\Omega} \operatorname{curl} \mathcal{A}_{2} f(x) \cdot \operatorname{curl} \psi(x) d x=\int_{\Omega} f(x) \cdot \psi(x) d x \neq 0$.

The Spectral Theorem for compact self-adjoint operators now yields an orthonormal basis $\left\{w_{j}\right\}_{j \in \mathbb{N}}$ of $L_{\Sigma}^{2}\left(\Omega, \mathbb{R}^{3}\right)$ and corresponding strictly positive eigenvalues $\mu_{j} \rightarrow 0$. We denote $\tilde{\lambda}_{j}:=1 / \mu_{j} \rightarrow \infty$. Equality A.8 implies that the mappings $w_{j} / \sqrt{\tilde{\lambda_{j}}}$ form an orthonormal system in $W_{\sigma}^{1,2}\left(\Omega, \mathbb{R}^{3}\right)$.

When $u \in W_{0, \sigma}^{1,2}\left(\Omega, \mathbb{R}^{3}\right), b \in W_{\sigma}^{1,2}\left(\Omega, \mathbb{R}^{3}\right)$ and $n \in \mathbb{N}$, Lemmas A.3 and A.6 allow us to write $P_{n} u$ and $Q_{n} b$ as

$$
P_{n} u=\sum_{j=1}^{n}\left(u, \frac{v_{j}}{\sqrt{\lambda_{j}}}\right)_{W_{0, \sigma}^{1,2}} \frac{v_{j}}{\sqrt{\lambda_{j}}}, \quad Q_{n} b=\sum_{j=1}^{n}\left(b, \frac{w_{j}}{\sqrt{\tilde{\lambda}_{j}}}\right)_{W_{\sigma}^{1,2}} \frac{w_{j}}{\sqrt{\tilde{\lambda}_{j}}} .
$$

This immediately implies the following result, which in turn yields the norm bound in A.6.

Proposition A.7. Both of the linear operators $P_{n}: W_{0, \sigma}^{1,2}\left(\Omega, \mathbb{R}^{3}\right) \rightarrow W_{0, \sigma}^{1,2}\left(\Omega, \mathbb{R}^{3}\right)$ and $Q_{n}: W_{\sigma}^{1,2}\left(\Omega, \mathbb{R}^{3}\right) \rightarrow W_{\sigma}^{1,2}\left(\Omega, \mathbb{R}^{3}\right)$ are self-adjoint and bounded uniformly in $n$.

In the next subsection we give a solution of the Galerkin approximation equations. 
A.2. The Galerkin approximation. In order to smoothen the exposition we work with the bases constructed in the previous subsection, although the following lemma holds for any orthonormal bases $\left\{v_{j}\right\}_{j \in \mathbb{N}}$ and $\left\{w_{j}\right\}_{j \in \mathbb{N}}$ of $L_{\sigma}^{2}\left(\Omega, \mathbb{R}^{3}\right)$ with $v_{j} \in W_{0, \sigma}^{1,2}\left(\Omega, \mathbb{R}^{3}\right)$ and $w_{j} \in W_{\sigma}^{1,2}\left(\Omega, \mathbb{R}^{3}\right)$.

Lemma A.8. For every $n \in \mathbb{N}$, the Galerkin approximation has a solution of the form A.1 with the energy equality A.2 holding for all $t \in[0, T)$.

Proof. When $u_{n}$ and $b_{n}$ are of the form A.1. Lemmas A.3 and A.6 imply that the Galerkin equations read as

$$
\dot{c}_{n j}(t)-\nu \lambda_{j} c_{n j}(t)+\sum_{k, l=1}^{n} c_{n k}(t) c_{n l}(t) \alpha_{j k l}-\sum_{k, l=1}^{n} d_{n k}(t) d_{n l}(t) \beta_{j k l}=0,
$$

$$
\dot{d}_{n j}(t)-\mu \tilde{\lambda}_{j} \chi_{j>N} d_{n j}(t)+\sum_{k, l=1}^{n} c_{n k}(t) d_{n l}(t) \gamma_{j k l}-\sum_{k, l=1}^{n} d_{n k}(t) c_{n l}(t) \delta_{j k l}=0,
$$

$$
c_{n j}(0)=\int_{\Omega} u_{0}(x) \cdot v_{j}(x) d x, \quad d_{n j}(0)=\int_{\Omega} b_{0}(x) \cdot w_{j}(x) d x
$$

for $j=1, \ldots, n$, where

$$
\begin{aligned}
\alpha_{j k l} & :=\int_{\Omega}\left(v_{k}(x) \cdot \nabla\right) v_{l}(x) \cdot v_{j}(x) d x, \quad \beta_{j k l}:=\int_{\Omega}\left(w_{k}(x) \cdot \nabla\right) w_{l}(x) \cdot v_{j}(x) d x \\
\gamma_{j k l} & :=\int_{\Omega}\left(w_{k}(x) \cdot \nabla\right) v_{l}(x) \cdot w_{j}(x) d x, \quad \delta_{j k l}:=\int_{\Omega}\left(v_{k}(x) \cdot \nabla\right) w_{l}(x) \cdot w_{j}(x) d x .
\end{aligned}
$$

Note that A.10 - A.12 is an initial value problem for a system of $2 n$ ODE's on the $2 n$ functions $c_{n j}, d_{n j}$, and by standard theory of ODE's there exists $T_{n}>0$ and a solution $c_{n 1}, \ldots, d_{n n} \in C^{\infty}\left(\left[0, T_{n}\right)\right)$. Note also that

$$
\alpha_{j k l}=-\alpha_{l k j}, \quad \beta_{j k l}=-\gamma_{l k j}, \quad \delta_{j k l}=-\delta_{l k j}=0 .
$$

The energy equality can be written as

$$
\begin{aligned}
& \sum_{j=1}^{n} c_{n j}(t)^{2}+\sum_{j=1}^{n} d_{n j}(t)^{2}+2 \sum_{j=1}^{n} \int_{0}^{t}\left(\nu \lambda_{j} c_{n j}(\tau)^{2}+\mu \tilde{\lambda}_{j} \chi_{j>N} d_{n j}(\tau)^{2}\right) d \tau \\
= & \sum_{j=1}^{n} c_{n j}(0)^{2}+\sum_{j=1}^{n} d_{n j}(0)^{2}
\end{aligned}
$$

and is proved by multiplying A.10 by $c_{n j}(t)$ and A.11) by $d_{n j}(t)$, summing in $j$, integrating in time and using (A.13). The energy equality allows us to continue the solution to A.10 A.12 to the whole interval $[0, T)$.

A.3. Passing to the limit. The Leray-Hopf solution $(u, b)$ of $(1.1)-(1.6)$ will be obtained as a strong $L^{2}$ limit of $\left(u_{n}, b_{n}\right)$ by using the Aubin-Lions Lemma. To that end we prove norm bounds on $\left(u_{n}, b_{n}\right)$.

Lemma A.9. There exists $C>0$ such that

$$
\begin{gathered}
\left\|u_{n}\right\|_{L^{\infty}\left(0, T ; L^{2}(\Omega)\right)}+\left\|u_{n}\right\|_{L^{2}\left(0, T ; W^{1,2}(\Omega)\right)}+\left\|\partial_{t} u_{n}\right\|_{L^{4 / 3}\left(0, T ;\left(W_{0, \sigma}^{1,2}(\Omega)\right)^{*}\right)} \leq C, \\
\left\|b_{n}\right\|_{L^{\infty}\left(0, T ; L^{2}(\Omega)\right)}+\left\|b_{n}\right\|_{L^{2}\left(0, T ; W^{1,2}(\Omega)\right)}+\left\|\partial_{t} b_{n}\right\|_{L^{4 / 3}\left(0, T ;\left(W_{\sigma}^{1,2}(\Omega)\right)^{*}\right)} \leq C
\end{gathered}
$$


for all $n \in \mathbb{N}$.

Proof. First, since $u_{n}$ and $b_{n}$ satisfy the energy equality for every $n \in \mathbb{N}, \| P_{n} u_{0}-$ $u_{0} \|_{L^{2}} \rightarrow 0$ and $\left\|Q_{n} b_{0}-b_{0}\right\|_{L^{2}} \rightarrow 0$, it follows that $\sup _{n \in \mathbb{N}}\left(\left\|u_{n}\right\|_{L^{\infty}\left(0, T ; L^{2}(\Omega)\right)}+\right.$ $\left.\left\|b_{n}\right\|_{L^{\infty}\left(0, T ; L^{2}(\Omega)\right)}\right)<\infty$. By another use of the energy equality and Lemma A.4. $\left|\nabla u_{n}\right|$ and $\left|\nabla b_{n, \Sigma}\right|$ are uniformly bounded in $L^{2}\left(0, T ; L^{2}(\Omega)\right)$. Furthermore,

$$
\begin{aligned}
\left\|\nabla b_{n, H}\right\|_{L^{2}\left(0, T ; L^{2}\left(\Omega, \mathbb{R}^{3 \times 3}\right)\right)} & =\left\|\sum_{i=1}^{\min n, N} d_{n j} \nabla w_{j}\right\|_{L^{2}\left(0, T ; L^{2}\left(\Omega, \mathbb{R}^{3 \times 3}\right)\right)} \\
& \lesssim \Omega\left\|\sum_{i=1}^{\min n, N} d_{n j} w_{j}\right\|_{L^{2}\left(0, T ; L^{2}\left(\Omega, \mathbb{R}^{3 \times 3}\right)\right)} \\
& =\left\|b_{n, H}\right\|_{L^{2}\left(0, T ; L^{2}\left(\Omega, \mathbb{R}^{3 \times 3}\right)\right)}
\end{aligned}
$$

for all $n \in \mathbb{N}$, and thus $\sup _{n \in \mathbb{N}}\left(\left\|u_{n}\right\|_{L^{2}\left(0, T ; W^{1,2}(\Omega)\right)}+\left\|b_{n}\right\|_{L^{2}\left(0, T ; W^{1,2}(\Omega)\right)}\right)<\infty$.

We now deal with $\partial_{t} b_{n}, \partial_{t} u_{n}$ being similar but slightly simpler. At a.e. $t \in[0, T)$ and for all $\theta \in W_{\sigma}^{1,2}\left(\Omega, \mathbb{R}^{3}\right)$ we write

$$
\left\langle\partial_{t} b_{n}, \theta\right\rangle_{\left(W_{\sigma}^{1,2}(\Omega)\right)^{*}-W_{\sigma}^{1,2}(\Omega)}+\left\langle\mu \Lambda_{2} b_{n}+\operatorname{curl}\left(b_{n} \times u_{n}\right), Q_{n} \theta\right\rangle_{\left(W_{\sigma}^{1,2}(\Omega)\right)^{*}-W_{\sigma}^{1,2}(\Omega)}=0 .
$$

Proposition A.7 gives

$$
\begin{aligned}
\left\langle\mu \Lambda_{2} b_{n}, Q_{n} \theta\right\rangle_{\left(W_{\sigma}^{1,2}(\Omega)\right)^{*}-W_{\sigma}^{1,2}(\Omega)} & =\mu\left(\operatorname{curl} b_{n}(\cdot, t), \operatorname{curl} Q_{n} \theta\right)_{L^{2}} \\
& \lesssim \mu\left\|b_{n}(\cdot, t)\right\|_{W_{\sigma}^{1,2}(\Omega)}\|\theta\|_{W_{\sigma}^{1,2}(\Omega)} .
\end{aligned}
$$

By using Proposition A.7 again, given $n \in \mathbb{N}$ and $\theta \in W_{\sigma}^{1,2}\left(\Omega, \mathbb{R}^{3}\right)$ we get

$$
\begin{aligned}
\left|\left\langle\operatorname{curl}\left(b_{n} \times u_{n}\right), Q_{n} \theta\right\rangle_{\left(W_{\sigma}^{1,2}(\Omega)\right)^{*}-W_{\sigma}^{1,2}(\Omega)}\right| & \leq\left\|b_{n}(\cdot, t)\right\|_{L^{6}}\left\|u_{n}(\cdot, t)\right\|_{L^{3}}\left\|\operatorname{curl} Q_{n} \theta\right\|_{L^{2}} \\
& \lesssim \Omega\left\|\nabla b_{n}(\cdot, t)\right\|_{L^{2}}\left\|u_{n}(\cdot, t)\right\|_{L^{3}}\|\theta\|_{W_{\sigma}^{1,2}},
\end{aligned}
$$

so that, by using the previous inequality and Hölder's inequality with exponents $3 / 2$ and 3 in $t$,

$$
\begin{aligned}
\left\|\operatorname{curl}\left(u_{n} \times b_{n}\right)\right\|_{L^{4 / 3}\left(0, T ;\left(W_{0, \sigma}^{1,2}\left(\Omega, \mathbb{R}^{3}\right)\right)^{*}\right)} & \lesssim \Omega\|\| \nabla b_{n}\left\|_{L^{2}\left(\Omega, \mathbb{R}^{3}\right)}\right\| u_{n}\left\|_{L^{3}\left(\Omega, \mathbb{R}^{3}\right)}\right\|_{L^{4 / 3}(0, T)} \\
& \leq\left\|\nabla b_{n}\right\|_{L^{2}\left(0, T ; L^{2}\left(\Omega, \mathbb{R}^{3 \times 3}\right)\right)}\left\|u_{n}\right\|_{L^{4}\left(0, T ; L^{3}\left(\Omega, \mathbb{R}^{3}\right)\right)}
\end{aligned}
$$

which, when combined with Lemma 2.11, completes the proof.

The Aubin-Lions Lemma and interpolation give various convergence properties.

Lemma A.10. There exist $u \in L^{\infty}\left(0, T ; L_{\sigma}^{2}\left(\Omega, \mathbb{R}^{3}\right)\right) \cap L^{2}\left(0, T ; W_{0, \sigma}^{1,2}\left(\Omega, \mathbb{R}^{3}\right)\right)$ and $b \in L^{\infty}\left(0, T ; L_{\sigma}^{2}\left(\Omega, \mathbb{R}^{3}\right)\right) \cap L^{2}\left(0, T ; W_{\sigma}^{1,2}\left(\Omega, \mathbb{R}^{3}\right)\right)$ such that, up to a subsequence, the following convergences hold:

(i) $u_{n} \rightarrow u$ and $b_{b} \rightarrow b$ in $L^{2}\left(0, T ; L_{\sigma}^{2}\left(\Omega, \mathbb{R}^{3}\right)\right)$,

(ii) $u_{n} \rightarrow u$ in $L^{2}\left(0, T ; W_{0, \sigma}^{1,2}\left(\Omega, \mathbb{R}^{3}\right)\right)$ and $b_{n} \rightarrow b$ in $L^{2}\left(0, T ; W_{\sigma}^{1,2}\left(\Omega, \mathbb{R}^{3}\right)\right)$,

(iii) $\partial_{t} u_{n} \rightarrow \partial_{t} u$ in $L^{4 / 3}\left(0, T ;\left(W_{0, \sigma}^{1,2}\left(\Omega, \mathbb{R}^{3}\right)^{*}\right)\right.$ and furthermore $\partial_{t} b_{n} \rightarrow \partial_{t} b$ in $L^{4 / 3}\left(0, T ;\left(W_{\sigma}^{1,2}\left(\Omega, \mathbb{R}^{3}\right)^{*}\right)\right.$,

(iv) $u_{n} \otimes u_{n} \rightarrow u \otimes u$ and $b_{n} \otimes b_{n} \rightarrow b \otimes b$ in $L^{4 / 3}\left(0, T ; L^{2}\left(\Omega, \mathbb{R}^{3 \times 3}\right)\right), b_{n} \times u_{n} \rightarrow$ $b \times u$ in $L^{4 / 3}\left(0, T ; L^{2}\left(\Omega, \mathbb{R}^{3}\right)\right)$.

Proof. While (i) and (ii) follow immediately from the Aubin-Lions Lemma and Lemma A.9, claims (iii)-(iv) follow from (i) and Lemma 2.11. The claim $u, b \in$ 
$L^{\infty}\left(0, T ; L_{\sigma}^{2}\left(\Omega, \mathbb{R}^{3}\right)\right)$ follows from the fact that up to a subsequence, $u_{n} \stackrel{*}{\rightarrow} u$ and $b_{n} \stackrel{*}{\rightarrow} b$ in $L^{\infty}\left(0, T ; L_{\sigma}^{2}\left(\Omega, \mathbb{R}^{3}\right)\right)$.

We show that $(u, b)$ solves the equations $\sqrt{1.1}-(5.3)$ and $(1.5)-(1.6)$, and we refer to 24 for the proof of the claims that $u, b \in C_{w}\left([0, T) ; L_{\sigma}^{2}\left(\Omega, \mathbb{R}^{3}\right)\right)$ and that $u(\cdot, 0)=u_{0}$ and $b(\cdot, 0)=b_{0}$. The energy inequality is then obtained as a consequence.

Lemma A.11. The mappings $u$ and $b$ mentioned in Lemma A.10 form a LerayHopf solution of (1.1)-(1.6).

Proof. We first show that $u$ and $b$ satisfy (2.3) and (2.4) a.t a.e. $t \in[0, T)$ for every $\varphi \in W_{0, \sigma}^{1,2}\left(\Omega, \mathbb{R}^{3}\right)$ and $\theta \in W_{\sigma}^{1,2}\left(\Omega, \mathbb{R}^{3}\right)$. Note that whenever $\eta \in C_{c}^{\infty}([0, T))$ and $k \in \mathbb{N}$, Lemmas A.8 and A.10 give

$$
\begin{aligned}
0 & =-\eta(0)\left(P_{n} u_{0}, v_{k}\right)_{L^{2}}-\int_{0}^{T} \eta^{\prime}(t)\left(u_{n}, v_{k}\right)_{L^{2}} d t \\
& -\int_{0}^{T} \eta(t)\left(u_{n} \otimes u_{n}-b_{n} \otimes b_{n}, \nabla v_{k}\right)_{L^{2}} d t+\nu \int_{0}^{T} \eta(t)\left(\nabla u_{n}, \nabla v_{k}\right)_{L^{2}} d t \\
& \rightarrow-\eta(0)\left(u_{0}, v_{k}\right)_{L^{2}}-\int_{0}^{T} \eta^{\prime}(t)\left(u, v_{k}\right)_{L^{2}} d t \\
& -\int_{0}^{T} \eta(t)\left(u \otimes u-b \otimes b, \nabla v_{k}\right)_{L^{2}} d t+\nu \int_{0}^{T} \eta(t)\left(\nabla u, \nabla v_{k}\right)_{L^{2}} d t,
\end{aligned}
$$

Given any $\varphi \in W_{0, \sigma}^{1,2}\left(\Omega, \mathbb{R}^{3}\right)$ we can replace $v_{k}$ above by $\varphi_{k}:=P_{k} \varphi$ by taking linear combinations. Now $\left\|P_{k} \varphi-\varphi\right\|_{L^{2}\left(\Omega, \mathbb{R}^{3}\right)} \rightarrow 0$ and $\sup _{k \in \mathbb{N}}\left\|P_{k} \varphi\right\|_{W_{0, \sigma}^{1,2}\left(\Omega, \mathbb{R}^{3}\right)}<\infty$ imply that $P_{k} \varphi \rightarrow \varphi$ in $W_{0, \sigma}^{1,2}\left(\Omega, \mathbb{R}^{3}\right)$. We let $k \rightarrow \infty$ to obtain

$$
\begin{aligned}
0 & =-\eta(0)\left(u_{0}, \varphi\right)_{L^{2}}-\int_{0}^{T} \eta^{\prime}(t)(u, \varphi)_{L^{2}} d t \\
& -\int_{0}^{T} \eta(t)(u \otimes u-b \otimes b, \nabla \varphi)_{L^{2}} d t+\nu \int_{0}^{T} \eta(t)(\nabla u, \nabla \varphi)_{L^{2}} d t,
\end{aligned}
$$

which in particular gives (2.3) at a.e. $t \in[0, T)$. Similarly, if $\theta \in W_{\sigma}^{1,2}\left(\Omega, \mathbb{R}^{3}\right)$, equation (2.4) holds at a.e. $t \in[0, T)$.

The claims that $u, b \in C_{w}\left([0, T) ; L_{\sigma}^{2}\left(\Omega, \mathbb{R}^{3}\right)\right)$ and that $u(\cdot, 0)=u_{0}$ and $b(\cdot, 0)=b_{0}$ can be proved by slightly modifying [24, Lemmas 2.1-2.2]. Since we have $u, b \in$ $C_{w}\left([0, T) ; L_{\sigma}^{2}\left(\Omega, \mathbb{R}^{3}\right)\right)$, it suffices to show the energy inequality at a.e. $t \in[0, T)$. Since $u_{n} \rightarrow u$ and $b_{n} \rightarrow b$ in $L^{2}\left(0, T ; L^{2}\left(\Omega, \mathbb{R}^{3}\right)\right)$, passing to a subsequence we get $u_{n}(\cdot, t) \rightarrow u(\cdot, t)$ and $b_{n}(\cdot, t) \rightarrow b(\cdot, t)$ in $L^{2}\left(\Omega, \mathbb{R}^{3}\right)$ at a.e. $t \in[0, T)$. At those times $t$ the energy inequality for $u$ and $b$ now follows from the energy equality of $u_{n}$ and $b_{n}$.

\section{REFERENCES}

[1] C. Amrouche, C. Bernardi, M. Dauge, and V. Girault, Vector potentials in three-dimensional non-smooth domains, Math. Methods Appl. Sci. 21 (1998), no. 9, 823-864.

[2] M. A. Berger, Rigorous new limits on magnetic helicity dissipation in the solar corona, Geophysical \& Astrophysical Fluid Dynamics 30 (1984), no. 1-2, 79-104.

[3] M. A. Berger and G. B. Field, The topological properties of magnetic helicity, J. Fluid Mech. 147 (1984), 133-148. 
[4] W. Borchers and H. Sohr, On the equations $\operatorname{rot} \mathbf{v}=\mathbf{g}$ and $\operatorname{div} \mathbf{u}=f$ with zero boundary conditions, Hokkaido Math. J. 19 (1990), no. 1, 67-87.

[5] A. C. Bronzi, M. C. Lopes Filho, and H. J. Nussenzveig Lopes, Wild solutions for 2D incompressible ideal flow with passive tracer, Commun. Math. Sci. 13 (2015), no. 5, 1333-1343.

[6] M. Brown, R. C. Canfield, and A.A. Pevtsov, Magnetic Helicity in Space and Laboratory Plasmas, Geophysical Monograph Series 111, AGU, 1999.

[7] T. Buckmaster, C. De Lellis, L. Székelyhidi, Jr., and V. Vicol, Onsager's conjecture for admissible weak solutions, arXiv:1701.08678 (2017).

[8] R. E. Caflisch, I. Klapper, and G. Steele, Remarks on Singularities, Dimension and Energy Dissipation for Ideal Hydrodynamics and MHD, Comm. Math. Phys. 184 (1997), 443-455.

[9] J. Cantarella, D. DeTurck, and H. Gluck, Vector calculus and the topology of domains in 3-space, Amer. Math. Monthly 109 (2002), no. 5, 409-442. MR 1901496

[10] J.-Y. Chemin, D. S. McCormick, J. C. Robinson, and J. L. Rodrigo, Local existence for the non-resistive MHD equations in Besov spaces, Adv. Math. 286 (2016), 1-31. MR 3415680

[11] R. Coifman, P.-L. Lions, Y. Meyer, and S. Semmes, Compensated compactness and Hardy spaces, J. Math. Pures Appl. 72 (1993), no. 3, 247-286.

[12] P. Constantin, W. E, and E. S. Titi, Onsager's conjecture on the energy conservation for solutions of Euler's equation, Comm. Math. Phys. 165 (1994), no. 1, 207-209.

[13] V. Dallas and A. Alexakis, The Signature Of Initial Conditions On Magnetohydrodynamic Turbulence, The Astrophysical Journal Letters 788 (2014), no. 2, 4 pp.

[14] J. I. Díaz and M. B. Lerena, On the inviscid and non-resistive limit for the equations of incompressible magnetohydrodynamics, Math. Models Methods Appl. Sci. 12 (2002), no. 10, $1401-1419$.

[15] J. M. de la Rasilla Dominguez, Etude des équations de la magnétohydrodynamique stationnaires et de leur approximation par éléments finis, Ph.D. thesis, Université de Paris VI, 1982.

[16] G. Duvaut and J.-L. Lions, Inéquations en thermoélasticité et magnétohydrodynamique, Arch. Rational Mech. Anal. 46 (1972), 241-279.

[17] G. L. Eyink, Dissipative anomalies in singular Euler flows, Phys. D 237 (2008), no. 14-17, 1956-1968.

[18] D. Faraco and S. Lindberg, Magnetic helicity and subsolutions in ideal MHD, arXiv:1801.04896v2 (2018).

[19] C. Fefferman and E. Stein, $H^{p}$ spaces of several variables, Act Math. 129 (1972), no. 3-4, $137-193$.

[20] C. L. Fefferman, D. S. McCormick, J. C. Robinson, and J. L. Rodrigo, Higher order commutator estimates and local existence for the non-resistive MHD equations and related models, J. Funct. Anal. 267 (2014), no. 4, 1035-1056. MR 3217057

[21] Local existence for the non-resistive MHD equations in nearly optimal Sobolev spaces, Arch. Ration. Mech. Anal. 223 (2017), no. 2, 677-691. MR 3590662

[22] J. M. Finn and T. M. Antonsen, Magnetic helicity: What is it and what is it good for?, Comments Plasma Phys. Controlled Fusion 9 (1985), no. 3, 111-126.

[23] C. Foiaş and R. Temam, Remarques sur les équations de Navier-Stokes stationnaires et les phénomènes successifs de bifurcation, Ann. Scuola Norm. Sup. Pisa Cl. Sci. (4) 5 (1978), no. $1,28-63$.

[24] G. P. Galdi, An introduction to the Navier-Stokes initial-boundary value problem, Fundamental directions in mathematical fluid mechanics, Adv. Math. Fluid Mech., Birkhäuser, Basel, 2000, pp. 1-70.

[25] _ An introduction to the mathematical theory of the Navier-Stokes equations, second ed., Springer Monographs in Mathematics, Springer, New York, 2011, Steady-state problems.

[26] J.-F. Gerbeau, C. Le Bris, and T. Lelièvre, Mathematical methods for the magnetohydrodynamics of liquid metals, Numerical Mathematics and Scientific Computation, Oxford University Press, Oxford, 2006.

[27] V. Girault and P.-A. Raviart, Finite element methods for Navier-Stokes equations, Springer Series in Computational Mathematics, vol. 5, Springer-Verlag, Berlin, 1986, Theory and algorithms.

[28] T. Hytönen, J. van Neerven, M. Veraar, and L. Weis, Analysis in Banach spaces. Vol. I. Martingales and Littlewood-Paley theory, Ergebnisse der Mathematik und ihrer Grenzgebiete. 3. Folge. A Series of Modern Surveys in Mathematics, vol. 63, Springer, Cham, 2016. 
[29] P. Isett, A Proof of Onsager's Conjecture, arXiv:1608.08301 (2016).

[30] E. Kang and J. Lee, Remarks on the magnetic helicity and energy conservation for ideal magneto-hydrodynamics, Nonlinearity 20 (2007), no. 11, 2681-2689.

[31] K. Kherief, Quelques propriétés des équations de la magnétohydrodynamique stationnaires et d'évolution, Ph.D. thesis, Université de Paris VII, 1984.

[32] H. Kozono and T. Yanagisawa, $L^{r}$-variational inequality for vector fields and the HelmholtzWeyl decomposition in bounded domains, Indiana Univ. Math. J. 58 (2009), no. 4, 1853-1920.

[33] J. Li, W. Tan, and Z. Yin, Local existence and uniqueness for the non-resistive MHD equations in homogeneous Besov spaces, Adv. Math. 317 (2017), 786-798. MR 3682684

[34] M. F. Linkmann, A. Berera, W. D. McComb, and M. E. McKay, Nonuniversality and finite dissipation in decaying magnetohydrodynamic turbulence, Physical Review Letters 114 (2015), no. 23.

[35] C. Miao and B. Yuan, Well-posedness of the ideal MHD system in critical Besov spaces, Methods Appl. Anal. 13 (2006), no. 1, 89-106. MR 2275873

[36] P. D. Mininni and A. Pouquet, Finite dissipation and intermittency in magnetohydrodynamics, Physical Review E 80 (2009), no. 2.

[37] C. B. Morrey, Jr., Multiple integrals in the calculus of variations, Die Grundlehren der mathematischen Wissenschaften, Band 130, Springer-Verlag New York, Inc., New York, 1966.

[38] J. Nečas, Direct methods in the theory of elliptic equations, Springer Monographs in Mathematics, Springer, Heidelberg, 2012, Translated from the 1967 French original by Gerard Tronel and Alois Kufner, Editorial coordination and preface by Šárka Nečasová and a contribution by Christian G. Simader. MR 3014461

[39] L. Onsager, Statistical hydrodynamics, Nuovo Cimento (9) 6 (1949), no. Supplemento, 2 (Convegno Internazionale di Meccanica Statistica), 279-287.

[40] J. C. Robinson, J. L. Rodrigo, and W. Sadowski, The three-dimensional Navier-Stokes equations, Cambridge Studies in Advanced Mathematics, vol. 157, Cambridge University Press, Cambridge, 2016, Classical theory.

[41] T. Roubíček, Nonlinear partial differential equations with applications, second ed., International Series of Numerical Mathematics, vol. 153, Birkhäuser/Springer Basel AG, Basel, 2013.

[42] P. G. Schmidt, On a magnetohydrodynamic problem of Euler type, J. Differential Equations 74 (1988), no. 2, 318-335. MR 952901

[43] P. Secchi, On the equations of ideal incompressible magnetohydrodynamics, Rend. Sem. Mat. Univ. Padova 90 (1993), 103-119. MR 1257135

[44] M. Sermange and R. Temam, Some mathematical questions related to the MHD equations, Comm. Pure Appl. Math. 36 (1983), no. 5, 635-664.

[45] J. B. Taylor, Relaxation of toroidal plasma and generation of reverse magnetic fields, Phys. Rev. Lett. 33 (1974), 1139-1141.

[46] _ Relaxation and magnetic reconnection in plasmas, Rev. Mod. Phys. 58 (1986), 741763

[47] R. Temam, Navier-Stokes equations, revised ed., Studies in Mathematics and its Applications, vol. 2, North-Holland Publishing Co., Amsterdam-New York, 1979.

[48] S. Wang, B. Wang, C. Liu, and N. Wang, Boundary layer problem and zero viscosity-diffusion limit of the incompressible magnetohydrodynamic system with no-slip boundary conditions, J. Differential Equations 263 (2017), no. 8, 4723-4749.

[49] J. Wu, Viscous and inviscid magnetohydrodynamics equations, J. Anal. Math. 73 (1997), $251-265$.

[50] Z. Wu and S. Wang, Zero viscosity and diffusion vanishing limit of the incompressible magnetohydrodynamic system with perfectly conducting wall, Nonlinear Anal. Real World Appl. 24 (2015), 50-60.

[51] Y. Xiao, Z. Xin, and J. Wu, Vanishing viscosity limit for the $3 D$ magnetohydrodynamic system with a slip boundary condition, J. Funct. Anal. 257 (2009), no. 11, 3375-3394.

[52] Z. Yoshida and Y. Giga, Remarks on spectra of operator rot, Math. Z. 204 (1990), no. 2, $235-245$

[53] J. Zhang, The inviscid and non-resistive limit in the Cauchy problem for 3-D nonhomogeneous incompressible magneto-hydrodynamics, Acta Math. Sci. Ser. B (Engl. Ed.) 31 (2011), no. 3, 882-896. MR 2830530 
Departamento de Matemáticas, Universidad Autónoma de Madrid, E-28049 Madrid, SPAIN; ICMAT CSIC-UAM-UC3M-UCM, E-28049 MADRID, SPAIN

E-mail address: daniel.faraco@uam.es

Departamento de Matemáticas, Universidad Autónoma de Madrid, E-28049 Madrid, SpAin; ICMAT CSIC-UAM-UC3M-UCM, E-28049 MADRID, Spain

E-mail address: sauli.lindberg@uam.es 